\title{
Rapid Prototyping of Microbial Cell Factories via Genome-scale Engineering
}

\author{
Tong $\mathrm{Si}^{\mathrm{a}, \dagger}$, Han Xiao ${ }^{\mathrm{a}, \dagger}$, and Huimin $\mathrm{Zhao}^{\mathrm{a}, \mathrm{b}, *}$
}

${ }^{a}$ Department of Chemical and Biomolecular Engineering, University of Illinois at Urbana-

Champaign, Urbana, IL 61801, 'bepartments of Chemistry, Biochemistry, and Bioengineering, Institute for Genomic Biology, University of Illinois at Urbana-Champaign, Urbana, IL 61801

${ }^{\dagger}$ These authors contributed equally to this work.

* To whom correspondence should be addressed. Phone: (217) 333-2631. Fax: (217) 333-5052.

E-mail: zhao5@illinois.edu 


\section{Abstract}

2 Advances in reading, writing and editing genetic materials have greatly expanded our ability to

3 reprogram biological systems at the resolution of a single nucleotide and on the scale of a whole

4 genome. Such capacity has greatly accelerated the cycles of design, build and test to engineer

5 microbes for efficient synthesis of fuels, chemicals and drugs. In this review, we summarize the

6 emerging technologies that have been applied, or are potentially useful for genome-scale

7 engineering in microbial systems. We will focus on the development of high-throughput

8 methodologies, which may accelerate the prototyping of microbial cell factories.

\section{Keywords}

11 Genome-scale engineering, microbial cell factory, transcriptome engineering, genome synthesis,

12 homologous recombination, high-throughput technology 


\section{Introduction}

14 Microbial cell factories (MCFs), which convert biomass resources to value-added compounds

15 such as fuels, chemicals, materials and pharmaceuticals, have been proposed as a sustainable and renewable alternative to the traditional petrochemical-based processes (Keasling, 2010, Lee et

17 al. , 2012, Rabinovitch-Deere et al. , 2013). However, intensive reprogramming of cellular metabolism is required to achieve economically feasible fermentation processes with MCFs. Conventional strain engineering approaches rely on random mutagenesis, which is achieved through chemical mutagens/UV irradiation (Crook and Alper, 2012), prolonged cultivation under selective pressure (Portnoy et al. , 2011), transposon insertions (Eckert et al. , 2011, Hamer et al. ,

22 2001, Hutchison et al. , 1999) and genome shuffling (Biot-Pelletier and Martin, 2014, Zhang et

23 al. , 2002). Effective in generating improved phenotypes using simple techniques, these methods

24 are widely adopted in industry, especially for those host organisms with poorly defined genetics

25 and limited engineering tools (Crook and Alper, 2012). However, traditional approaches are

26 often labor-intensive, time-consuming, and difficult to analyze and transfer the genetic basis of a

27 selected trait. Recently, the scale, efficiency and precision of genetic analysis and manipulation have been remarkably improved by several enabling technologies, including but not limited to microarray DNA synthesis, next-generation DNA sequencing (NGS), programmable DNAbinding proteins, and in vivo biosensors. Nowadays, billions of genome variants can be created

31 in a directed and/or combinatorial manner, and the mutant strains with the optimal performance 32 can be rapidly isolated. Collectively, these new technologies and their applications exemplify an emerging discipline called 'genome engineering' or 'genome-scale engineering' (Carr and 34 Church, 2009, Esvelt and Wang, 2013, Jeong et al. , 2013, Segal and Meckler, 2013). 
36 The practice of genome-scale engineering can be broadly classified into three categories: genome

37 editing, transcriptome engineering, and genome synthesis. Genome editing precisely or

38 combinatorially modifies the target genome at multiple loci. Modifications are located either in

39 the open-reading frames (ORFs) or in the cis-acting regulatory elements such as promoters and 40 ribosome-binding sites (RBSs). Transcriptome engineering essentially targets trans-acting

41 regulatory elements, such as transcription factors (TFs) or non-coding RNAs (ncRNAs), by 42 mutating endogenous regulators or introducing artificial ones. Genome synthesis involves

43 hierarchical assembly of short chemically synthesized DNA fragments into viral/microbial 44 genomes and yeast chromosomes. Although current synthetic genomes are constructed mainly 45 based on their wild type templates, the ultimate goal is to write genome sequences de novo.

47 In this review, we first introduce the recent development in genome editing (section 2), 48 transcriptome engineering (section 3), and genome synthesis (section 4). We then highlight how 49 these techniques can facilitate high-throughput genotyping and phenotyping (section 5), which 50 greatly accelerates our understanding and engineering of microbial genomes. In addition, we

51 will discuss several examples on the application of genome-scale engineering to improve MCF 52 performance and provide perspectives on how computational approaches and laboratory 53 automation can be further integrated.

\section{$55 \quad 2$ Genome editing}

56 Unlike random mutagenesis, targeted genome editing results in elaborative and massive genome 57 modifications with a traceable manner. Homologous recombination (HR) is the core mechanism 
58 of most targeted genome editing techniques, and various enzymes have therefore been

59 investigated to either mediate or promote HR in microorganisms.

\subsection{Recombinases}

62 Recombinases catalyze exchange of short homologous regions (30 40 bp) of DNA. Site63 specific recombinases are grouped into two families, the tyrosine recombinase family and the 64 serine recombinase family (Turan et al. , 2013). An early characterized member of the tyrosine

65 recombinase family was $\lambda$ integrase, which enables incorporation of phage DNA into the 66 bacterial chromosomes. The $\lambda$ integrase mediates irreversible recombination between the attP 67 and $a t t B$ sites in the phage and host chromosomes respectively, generating recombinant att $L$ and 68 attR sites (Mizuuchi and Mizuuchi, 1980). Later, Cre (from phage P1) and flippase (FLP, from 69 the $2 \mu$ plasmid of yeast Saccharomyces cerevisiae), recognizing the loxP site and the flippase 70 recognition target (FRT) site, respectively, were widely used for efficient recombination in a 71 variety of species (Dymecki, 1996, Nagy, 2000, Sternberg et al. , 1981, Turan et al. , 2011).

72 With identical recognition sites, Cre and FLP can reversibly invert, integrate or excise DNA 73 sequences between recognition regions. Alternatively, such processes can be made irreversible 74 using a partially mutated recognition site to yield a poorly recognized region after recombination 75 (Albert et al. , 1995, Schlake and Bode, 1994). For the serine recombinase family, $\phi C 31$ 76 integrase (from Streptomyces phage $\phi C 31$ ) was the most well-studied example. Behaving like 77 the $\lambda$ integrase, $\phi C 31$ was proven to have great potential in eukaryotic genome engineering 78 (Karow and Calos, 2011). 
80 In addition to inversion, integration and excision facilitated by the above-mentioned

81 recombinases, recombinase-mediated cassette exchange (RMCE) is another useful approach in

82 genome engineering. By flanking the target genomic locus with two different spacer mutant

83 ("heterospecific") sites recognized by the same recombinase or orthogonal sites of different

84 recombinases, the endogenous region will be replaced by a donor cassette with compatible

85 recognition sites (Turan, Zehe, 2013). Many Cre and FLP variants were engineered to recognize

86 different sites with little cross reactivity with the wild type system, allowing efficient directional

87 cassette exchange (Fig. 1A) (Buchholz and Stewart, 2001, Schlake and Bode, 1994, Turan et al. ,

88 2010). By exploiting the specific attP $\mathrm{x}$ attB recombination event, $\phi C 31$ was also applied to

89 cassette exchange without the requirement of heterospecific att-sites. However, $\phi \mathrm{C} 31$ mediated-

90 cassette change was in a unidirectional manner (Turan and Bode, 2011).

92 Notably, pre-existing recognition sites are required for all events mediated by recombinases.

93 Therefore, introduction of recognition sites into the target locus is unavoidable, which limits the 94 application of recombinase-based methods for genome editing. Although much effort has been

95 invested in the directed evolution of recombinases with new target recognition sequences, the 96 engineered enzymes were inefficient in most cases (Gordley et al. , 2009).

\subsection{Recombination-mediated genetic engineering (Recombineering)}

99 Taking advantage of bacteriophage-based recombination proteins (e.g., Red-Exo, Beta and Gam

100 from $\lambda$ phage (Murphy, 1998) and Rec E/T from Rac prophage (Zhang et al. , 1998)), 101 recombineering enables efficient and large-scale recombination between the transformed DNA 
102 fragments and the bacterial genome (Sharan et al. , 2009). Recombination through the $\lambda$ Red 103 system relies on three proteins: Exo (also known as $\alpha$ ), Beta and Gam. Exo is a 5'to 3' 104 exonuclease that digests double-stranded DNA (dsDNA) with 3' overhangs generated. Beta is a 105 single-stranded DNA (ssDNA) binding protein, which facilitates recombination between target 106 locus and donor DNA (Fig. 1B). Gam inhibits endogenous RecBCD and SbcCD activity, 107 preventing degradation of exogenous DNA by the host (Datta et al. , 2008). In the RecE/T 108 system, RecE is a 5'to 3' exonuclease (similar to Exo) and RecT is a ssDNA-binding protein that 109 stabilizes ssDNA and promotes recombination (similar to Beta). Either dsDNA or ssDNA can 110 be employed for recombineering (Ellis et al. , 2001, Sawitzke et al. , 2007). With a linear donor 111 DNA flanked by homology sequences as short as $40 \mathrm{bp}$, efficient recombination can be achieved 112 by the $\lambda$ red system (Sharan, Thomason, 2009). In addition, the RecET proteins are more 113 efficient than the $\lambda$ red proteins, especially in case of HR between two linear molecules (Fu et al., 114 2012). When these bacteriophage proteins were expressed in E. coli, the recombination 115 efficiencies dramatically increased from 1 per $10^{6}$ cells to 1 per $10^{3-4}$ cells without optimization 116 (Boyle et al. , 2013, Murphy, 1998, Swingle et al. , 2010, Zhang, Buchholz, 1998).

118 With further optimization (e.g., modifying the cellular machinery involved in DNA replication 119 (Lajoie et al. , 2012) or optimizing the concentration and length of donor oligos (Sawitzke et al. , 120 2011)), recombineering technology has been exploited for multiplexed genome engineering. 121 Notably, multiplex automated genome engineering (MAGE) and trackable multiplex 122 recombineering (TRMR) accelerated E. coli genome evolution and analysis by creating genome123 wide combinatorial genetic modifications in a short time (Wang et al. , 2009, Warner et al. , 124 2010). These studies are discussed in greater details in later sections. 


\subsection{Endonucleases}

127 Apart from recombineering, introduction of a double-strand break (DSB) to a chosen site is 128 another important strategy for targeted genome editing. Normally, a DSB is repaired by either 129 HR or non-homologous end joining (NHEJ). Given a donor sequence flanked by homologous 130 regions to target locus, HR can be facilitated by a DSB (Iglehart and Silver, 2009). In some 131 hosts with low HR efficiency (e.g., mammalian cells), error-prone NHEJ is boosted by DSBs, 132 which results in gene disruption (Sun et al. , 2012b). Taken together, DSB-promoted HR or 133 NHEJ eases the arduous targeted genome editing in most organisms. As a result, different 134 natural and artificial endonucleases have been developed and engineered to introduce DSBs at 135 desired loci (Fig. 1C).

\section{$137 \quad$ 2.3.1 I-SceI meganucleases}

I-SceI is a homing endonuclease from the mitochondria of Saccharomyces cerevisiae and was

139 the first endonuclease used in genome engineering (Silva et al., 2011). Compared to traditional 140 restriction enzymes, I-SceI recognizes a longer sequence (18 bp), rendering it more rare-cutting 141 (once in every $6.9 \times 10^{10} \mathrm{bp}$ ). Indeed, HR promoted by I-SceI has been demonstrated in several 142 prokaryotes and eukaryotes (Cox et al. , 2007, Maggert et al. , 2008, Yu et al. , 2008). However, 143 like recombinases, the requirement to introduce a recognition site into a target locus limits the 144 application of I-SceI-based methods. Directed evolution of I-SceI towards a pre determined site 145 was an alternative to make I-SceI more programmable. However, a substantial challenge arose 
146 since the DNA binding domain could not be decoupled from cleavage domain in naturally

147 occurring I-SceI (Moure et al. , 2008).

\subsubsection{Zinc-finger nucleases (ZFNs)}

150

151

152

153

154

155

156

157

158

159

160

161

162

163

164

165

166

ZFNs are artificial proteins that combine the DNA binding domain of a zinc finger protein with the non-specific cleavage domain of a FokI endonuclease, providing a general strategy to deliver site-specific DSBs to the chromosome (Li et al. , 1992, Pavletich and Pabo, 1991). A zinc finger (ZF) is a $\sim 30$ amino acid motif that recognizes 3 nucleotides of DNA. Three ZF repeats are typically contained in an individual ZFN (Pavletich and Pabo, 1991). To strengthen the interaction of two FokI cleavage domains, a pair of zinc fingers is designed to bind neighboring sequences with a 5 to $7 \mathrm{bp}$ spacer to form a dimeric cleavage domain. Such optimal configuration allows dimerization and subsequent cleavage (Bibikova et al. , 2001). Successful ZFN-induced genome modifications were reported in many organisms such as plants (Townsend et al. , 2009), zebrafish (Meng et al. , 2008), frogs (Young et al. , 2011), mice (Carbery et al. , 2010), and sea urchin (Ochiai et al. , 2010). Potential off-target effects were alleviated by utilizing a less toxic nickase (Kim et al. , 2012) or additional ZF repeats (four to six) to bind longer sites (Wood et al. , 2011). However, it has been shown that each triplet recognized by a ZF cannot be simply assembled to recognize a longer sequence (Ramirez et al. , 2008). As a result, synthesizing customized ZFs remains difficult and expensive (Carroll, 2011).

\subsubsection{Transcription activator-like effector nucleases (TALENs)}


167 Similar to ZFNs, TALENs fuse the DNA-binding domain of a transcription activator-like 168 effector (TALE) with a catalytic nuclease domain. TALE is a bacterial effector protein for 169 synergistic regulation of gene expression in Xanthamonas sp. and Ralstonia sp. (Fu et al. , 2013). 170 Highly conserved 33-35 amino acid TALE repeat domains each bind one nucleotide of DNA 171 with specificity dictated by two hypervariable residues (Fu, Foden, 2013). Unlike ZFNs, this 172 one-to-one code allows the design of proteins with desired DNA-binding specificities by simply 173 concatenating TALE repeats. Due to the more flexible recognition rule, TALENs can in 174 principle be designed to readily target any sequence across the genome. In fact, TALENs have 175 been applied in various organisms such as human cells (Sun et al., 2012c), yeast (Li et al. , 2011) 176 and zebrafish (Li, Huang, 2011). According to some preliminary studies, TALENs also seem to 177 have fewer off-target effects compared to the corresponding ZFNs (Mussolino et al. , 2011). 178 Nonetheless, the primary drawback of TALENs is the trickiness of assembling a large number of 179 repeats into an array. Several strategies have been reported to address this limitation (Briggs et 180 al. , 2012, Liang et al. , 2013).

\subsubsection{CRISPR nucleases}

The type II bacterial Clustered Regularly Interspaced Short Palindromic Repeats and CRISPR-

184 associated proteins (CRISPR-Cas) system has recently been exploited as an efficient gene185 targeting technology (Cong et al. , 2013, Hwang et al. , 2013, Jiang et al. , 2013, Wang et al. , 2013a). Directed by a trans-activating crRNA (tracrRNA):crRNA duplex (or a chimeric guide

187 RNA (gRNA)), the CRISPR nuclease (e.g., Cas9 protein) is able to cleave a target DNA 188 sequence with the protospacer adjacent motifs (PAM) (Mali et al. , 2013). The 12 bp of rigorous 189 homology at 5' of the PAM sequence ensured the activity of the CRISPR nuclease (Cong, Ran, 
190

191

192

193

194

195

196

197

198

199

200

201

202

203

204

205

206

207

208

209

210

211

2013). Diversified short PAM sequences (e.g., NGG (Deltcheva et al. , 2011), NAAR (van der Ploeg, 2009), NGGNG (Horvath et al. , 2008)) recognized by different CRISPR nucleases permit almost all sequences to be targeted. Rather than protein based recognition by ZFNs or TALENs, the nucleic acid-based recognition by CRISPR nucleases significantly eases the assembly process. CRISPR-Cas assisted genome modifications have been demonstrated in many species such as mammalian cells (Cong, Ran, 2013, Mali, Yang, 2013), yeast (Dicarlo et al. , 2013b), E. coli (Jiang et al. , 2013) and plants (Feng et al. , 2013). In some cases, the HR and NHEJ efficiencies mediated by the CRISPR nuclease were much higher than those obtained by TALENs (Mali, Yang, 2013). Morever, efficient multiple deletions were also achieved in mammalian cells and S. cerevisiae using CRISPR-Cas (Bao et al. , 2014, Wang, Yang, 2013a). However, because of the short recognition sequence (12 bp), many off-target cleavages may be generated by CRISPR nucleases, which may be a serious issue in hosts with large genome sizes. In fact, the off-target problem has been mitigated by several approaches in recent studies. With a pair of closely spaced Cas9 variants (Cas9-D10A) nicking adjacent regions on opposite DNA strands rather than introducing DSBs, the mutation frequencies of off-target sites were reduced in mammalian cells (Cho et al. , 2014, Shen et al. , 2014). To circumvent the difficulties of adopting the paired nicking strategy for multiplex or genome-scale targeting by CRISPR-Cas, Fu et al. successfully improved the targeting specificity using truncated gRNAs with 17 or 18 nucleotides. Most importantly, those truncated gRNAs can function as efficiently as (or even more efficiently than) their matched 20 nucleotides counterparts (Fu et al. , 2014).

\subsection{Group II introns}


212 A group II intron (also known as a targetron) consists of a self-catalytic RNA and a 213 ribonucleoprotein (RNP), which catalyzes the insertion of an intron into specific DNA sites via 214 retrohoming (Michel and Ferat, 1995) (Fig. 1D). Through base-pairing with the intron RNA, 215 site-specific insertions or disruptions can be accomplished by group II introns. Group II intron 216 based gene disruptions are prevalent in prokaryotes, albeit there are a few applications in 217 eukaryotes (Jeong, Cho, 2013). Group II introns have been used in bacterial hosts with fairly 218 low HR efficiency (e.g., Clostridia) (Shao et al. , 2007). In addition, group II introns can 219 promote targeted insertion, deletion, inversion and cassette exchange by delivery of recombinase 220 recognition sites (e.g., loxP sites) to a given locus (Enyeart et al. , 2013). Group II introns were 221 also reported to introduce site-specific DSBs (Karberg et al. , 2001), which may facilitate HR or 222 NHEJ-based DNA repair. As observed with other programmable nucleases (e.g., TALENs 223 (Aouida et al. , 2014) and CRISPR (Bao, Xiao, 2014)), the targeting efficiency mediated by 224 group II introns was also site-dependent (Perutka et al. , 2004).

\section{Transcriptome engineering}

227 In addition to genome editing, transcriptome engineering provides a complementary strategy for 228 genome-scale engineering. Targeting at trans-acting regulatory elements, genetic modulation is 229 achieved without modifying the target chromosomal loci. This feature eliminates the need for 230 prior knowledge of host genomes, which is required by most genome editing methods that 231 depend on homologous recombination. Transcription factors (TFs) and regulatory non-coding 232 RNAs (ncRNAs) are the most common targets for transcriptome engineering. 


\subsection{Transcription factor engineering}

235 Thanks to transcriptional regulatory networks, cells can rapidly coordinate the expression of 236 thousands of genes when facing both internal and environmental stimuli (Lopez-Maury et al. , 237 2008). Such networks exhibit pyramid-shaped hierarchical structures, with most transcription 238 factors (TFs) at the bottom and middle levels, and only a few master TFs on the top for global 239 regulation (Yu and Gerstein, 2006). Whereas specific TFs at the bottom levels modulate dozens 240 of genes in the same functional group, the master TFs have global influence over the gene 241 expression profile ( $\mathrm{Yu}$ and Gerstein, 2006). These features make TFs the ideal targets for 242 transcriptome reprogramming by modulating many genes simultaneously (Lin et al. , 2013, 243 Santos and Stephanopoulos, 2008). Two main strategies have been applied to engineer TFs: 244 modulation of native transcriptional machinery and introduction of artificial TFs (Fig. 1E).

246 As a demonstration of native TF engineering, global transcriptional machinery engineering 247 (gTME) introduces mutations to the master TFs that mainly mediate DNA recognition, based on 248 the assumption that variations in these TFs may exert substantial changes to the promoter 249 preference of the RNA polymerase. As proof of concept, the principal sigma factor in E. coli

$250\left(\sigma^{70}\right)$ was subjected to error-prone PCR. From the resultant strain libraries, mutants with 251 improved tolerance to sodium dodecyl sulfate (SDS) and ethanol were identified through serial 252 subculturing (Alper and Stephanopoulos, 2007). In S. cerevisiae, the TATA-binding protein 253 Spt15p and a TATA-binding protein-associated factor Taf25p were mutated. The best variant, 254 which harbored three amino acid mutations in Spt15p, conferred a 70\% improvement in ethanol 255 productivity (Alper et al. , 2006). It has also been demonstrated that gTME is more effective in 256 diversity creation than chemical mutagenesis methods, and therefore increases the possibility to 
257 isolate phenotypes that were unattainable through traditional methods (Klein-Marcuschamer and 258 Stephanopoulos, 2008).

On the other hand, artificial transcription factor (ATF) libraries have also been created to 261 generate transcriptional diversities. A minimal ATF may only contain a DNA-binding domain, 262 whose interaction with its target sequence most likely down-regulates the expression of a nearby 263 gene by interfering with transcriptional initiation or elongation (Park et al. , 2005). The DNA264 binding domain can also be attached to effector (activator/repressor) domains or ligand-binding 265 domains, which permits more sophisticated regulation. Most ATFs reported so far have 266 employed zinc finger proteins (ZFPs) as the DNA-binding domains, and the library of ATFs is 267 constructed through combinatorial assembly of individual zinc fingers with diverse DNA268 binding specificities. The first example of such effort was the introduction of over $10^{5}$ ZFPs 269 fused with effector domains into S. cerevisiae (Park et al. , 2003). The library consisted of three270 or four-finger ZFPs, which recognize 9 bp or 12 bp DNA sequences with limited randomness 271 constrained by choice of individual zinc fingers (40 and 25 individual zinc fingers for three- and 272 four-finger proteins, respectively). Several ATFs were identified to confer a number of tolerance 273 phenotypes towards heat, osmotic pressure and an antifungal drug ketoconazole. The relatively 274 short recognition sequence (9 bp or $12 \mathrm{bp}$ ) permits the ATFs to modulate many genes. For 275 example, one selected artificial transcriptional factor (K7), which conferred ketoconazole 276 resistance, had 14 perfectly matched binding sites in the yeast genome (Park, Lee, 2003). The 277 perturbation scope by these AFTs may be even larger considering off-target effects. A similar 278 strategy has been applied to E. coli to isolate tolerant strains towards heat shock (Park, Jang, 2792005 ) and butanol (Lee et al. ,2011). For future development, we envision that a new generation 
280 of ATFs can be developed when the DNA-binding domains are changed from ZFPs to TALEs

281 and CRISPR proteins. As the DNA-binding specificity of TALEs and CRISPR proteins is much

282 more predictable than that of ZFPs, transcriptome perturbation by TALE- and CRISPR-derived

283 ATF libraries should be more effective and programmable.

\subsection{Regulatory non-coding RNAs}

286 Non-coding RNA molecules (ncRNAs) are increasingly recognized as key regulators across the

287 biological kingdoms (Kang et al. , 2014, Qi and Arkin, 2014). Here we will mainly focus on 288 regulatory ncRNAs that have been used in genome-scale engineering (Fig. 1F). To be suitable 289 for genome-wide applications, synthetic ncRNAs should be preferably trans-acting, permitting 290 simple introduction of a genome-wide library with minimal considerations on local genetic 291 context. Also, the interaction between an ncRNA and its DNA or mRNA target should be 292 mainly determined by Watson-Crick base pairing, so that the binding specificity and efficiency 293 can be predictable and programmable.

In bacteria, trans-acting small RNAs (sRNAs) and antisense RNAs (asRNAs) are two main

296 regulatory ncRNAs (Qi and Arkin, 2014). Lee and coworkers recently developed a general 297 framework to design synthetic sRNAs in E. coli for metabolic engineering (Na et al. , 2013). 298 The synthetic sRNAs were composed of a scaffold sequence and a target-binding sequence. The 299 scaffold was derived from a naturally occurring sRNA, MicC, and the scaffold can recruit the 300 Hfq protein to facilitate sRNA-mRNA interaction and mRNA degradation. The native target 301 binding sequence of $M i c C$ can be replaced by the antisense sequence to the translation initiation 
302 region (TIR) of any given gene. Correlation was found between the repression capability and the

303 binding energy of the antisense sequence, which allowed for fine-tuning of the knockdown

304 efficiency. Although the sRNA library constructed in that study only targeted the cadaverine

305 production related genes (Na, Yoo, 2013), it is possible to expand the strategy to a genome-scale.

307 On the other hand, asRNAs have been used for functional genomics study in a series of bacteria, 308 such as Streptococcus mutans (Wang and Kuramitsu, 2005) and Staphylococcus aureus (Forsyth 309 et al. , 2002). However, it has been long recognized that asRNAs are inefficient for gene 310 repression in E. coli (Wagner and Flardh, 2002). Recently, it was found that asRNA molecules 311 with paired-termini have enhanced stability and improved repression capacity (Nakashima et al. , 312 2006). E. coli genomic DNA fragments have been cloned into a paired-termini expression vector 313 to generate a genome-wide asRNA library, which was used to successfully isolate asRNAs that 314 target essential genes and led to conditional growth inhibition (Meng et al. , 2012).

316 As for eukaryotes, the most common ncRNA machinery for gene expression regulation is RNA 317 interference (RNAi), a cellular gene silencing mechanism whereby mRNAs are targeted for 318 degradation by homologous double-stranded RNAs (dsRNAs) (Fire et al. , 1998, Hannon, 2002). 319 RNAi proves to be a powerful tool for genome-wide reduction-of-function screen in many higher 320 eukaryotes (Boutros and Ahringer, 2008, Echeverri and Perrimon, 2006), yet its applications in 321 microbes are rare. This is probably due to the lack of a native RNAi pathway in S. cerevisiae, 322 which is the most-widely used microbial eukaryote. Recently, a heterologous RNAi machinery 
323 has been reconstituted in S. cerevisiae (Drinnenberg et al. , 2009), which opens up the possibility

324 of genome-wide RNAi screen (see Section 6.3).

326 In addition to naturally-occurring ncRNAs, synthetic RNAs were also used to modulate gene 327 expression in the CRISPR-mediated interference (CRISPRi) and CRISPR-mediated activation 328 technologies (Fig. 1F). By co-expressing of a Cas9 mutant with abolished endonuclease activity 329 (dCas9) and a guide RNA targeting at the non-template DNA strand of a target gene, up to 330 1,000-fold reduction in gene expression was achieved in E. coli (Qi et al. , 2013). In $S$. 331 cerevisiae, the silencing efficiency may be further improved by fusing the dCas9 protein to 332 transcription repressors or chromatin silencers (Gilbert et al. , 2013). For gene activation, 333 transcriptional activators can be delivered by the dCas9-guide RNA complex to the upstream 334 region of a promoter, resulting in up-regulation in E. coli (Bikard et al. , 2013) and yeast 335 (Farzadfard et al. , 2013, Gilbert, Larson, 2013).

\section{4. Genome synthesis}

338 Genome synthesis is one of the most impressive achievements of synthetic biology, ranging from 339 viral genomes (Cello et al. , 2002, Chan et al. , 2005) and bacterial genomes (Gibson et al. , 2008, 340 Gibson et al. , 2010, Karas et al. , 2012) to yeast chromosomes (Annaluru et al. , 2014, Dymond 341 et al. , 2011). Early efforts mainly focused on increasing the scale of the final DNA constructs, 342 from the $7.5 \mathrm{~kb}$ cDNA copy of a poliovirus genome (Cello, Paul, 2002) to a $1.08 \mathrm{Mb}$ bacterial 343 genome (Gibson, Glass, 2010). In terms of DNA sequences, the synthetic genomes are almost 344 exact copies of the native ones, except for a few inserted "watermarks" such as the names of the 
345 team members (Gibson, Benders, 2008, Gibson, Glass, 2010). A recent report took a step further

346 to build a designer yeast chromosome that was substantially different from its wild-type template

347 (Annaluru, Muller, 2014). Compared to the native chromosome III of S. cerevisiae, the designer

348 chromosome synIII was $\sim 14 \%$ smaller due to the deletion of some non-essential regions such as

349 transfer RNAs, transposons and introns. However, the design principles are still very simplistic,

350 and it requires substantial technological development before we can write a fully synthetic

351 genome. Therefore, rather than discussing the practice of genome synthesis, we will instead

352 focus on its enabling technologies, such as DNA synthesis and parts engineering-and code

353 expansion, as these technologies are also highly useful in engineering better microbial cell

354 factories.

356 4.1. DNA synthesis

357 One fundamental enabling factor for synthetic genomes is the decreasing cost of chemical DNA 358 synthesis in a manner akin to Moore's law (Mueller et al. , 2009). While further reduction of the 359 synthesis cost using the traditional column-based methods is unlikely, microarray-based 360 technologies provide great potential for high-throughput and cost-effective DNA synthesis, as 361 discussed in details elsewhere (Mueller, Coleman, 2009, Tang et al. , 2013). Here we will focus 362 on two recent advances in microarray-based DNA synthesis, highlighting the importance of 363 technology integration. One major limitation of chip-synthesized sequences is that they are 364 prone to error. In addition to the optimization of the microarray technology itself, the integration 365 of next-generation sequencing (NGS) has greatly improved the fidelity of synthetic DNA 366 (Matzas et al. , 2010). A pool of chip-derived oligonucleotides were attached to individual 367 streptavidin-coated beads and amplified via emulsion PCR. Pyrosequencing was performed, and 
368 the beads with correct sequences were sorted in a high-throughput manner by a camera-guided

369 micropipette. The fidelity was estimated to be improved by 500 -fold, and the throughput may

370 potentially enable DNA construction at a megabase scale (Matzas, Stahler, 2010). Another

371 challenge lies at the heterogeneity of the microarray-generated oligo pools, as well as the limited

372 amount of DNA synthesized at an individual spot, both of which complicate the subsequent gene

373 assembly. Quan et al. devised an integrated solution to overcome this challenge, by combining

374 the synthesis, amplification and assembly steps (Quan et al. , 2011). The microchip was divided

375 into microfluidics aided subarray reactors, each containing oligonucleotides for the assembly of

376 the same gene. After synthesis, the oligos were amplified, released and assembled into gene

377 constructs up to $1 \mathrm{~kb}$ each by enzymatic reactions. Together, both methods discussed above

378 demonstrate that novel solutions can be achieved through technology integration, which will

379 continue to be a driving force to provide synthetic DNA with lower price and higher quality.

\section{$381 \quad$ 4.2. Parts engineering}

382 With the DNA synthesis capacity becoming less restrictive, the limited repertoire of genetic parts 383 and a lack of design principles are becoming the two major obstacles in synthetic genome 384 construction (Wang et al. , 2013b). Rather than providing comprehensive summary on how to 385 overcome these obstacles, we will only focus on the expansion of part collections, which also 386 provides sources for diversity generation to improve MCF performance. The readers are directed 387 to other excellent reviews for the design framework for functional assembly of biological parts 388 (Wang, Wei, 2013b, Way et al. , 2014), as well as experimental protocols for physical assembly 389 of DNA fragments (Chao et al. , 2014). 
391 Based on their functional roles, genetic parts can be classified as sensors (take in environmental

392 stimuli, e.g. riboswitches), regulators (perform calculation, e.g. promoters), actuators (generate 393 outputs, e.g. structural proteins) and adapters (connect components, e.g. endoplasmic reticulum 394 (ER) tags) (Wang, Wei, 2013b). Here we will mainly discuss transcriptional regulators, such as 395 promoters, RBSs and terminators, as they are so far still the most available, well-studied and 396 widely-used genetic parts. Given the wide application of microbial biotechnology, the most 397 important task on these building blocks is to discover, engineer and characterize as many 398 biological parts as possible (Wang, Wei, 2013b). There are mainly three methods to enlarge the 399 parts collection: to harvest from nature, to create mutant libraries, and to build by modeling.

401 Advances in sequencing and bioinformatics permit rapid identification and prototyping of 402 biological parts from genome and megagenome sequences. For example, strong constitutive 403 promoter candidates can be isolated from the upstream sequences of housekeeping genes (such 404 as global transcription/translation factors, glycolytic enzymes, etc) (Shao et al. , 2013, Sun et al. , 405 2012a). High-throughput techniques may also allow characterization of all putative promoters in 406 a microbe (Zaslaver et al. , 2006). To create a mutant library of biological parts, there are 407 essentially three strategies. First, mutations can be introduced via error-prone PCR, and the 408 resultant variants with desirable performance (e.g. various promoter strengths) are isolated 409 through high-throughput methods (Alper et al. , 2005, Nevoigt et al. , 2006). Second, the 410 heterogeneous DNA oligonucleotide pools synthesized by microarray can serve as a source for 411 diversity. For example, synthetic intergenic regions were combinatorially assembled from three 412 oligo libraries with overlapping ends, modulating relative expression patterns of multiple 
413 enzymes in a synthetic operon (Pfleger et al. , 2006). Also, recombineering enabled the

414 replacement of the native ribosome binding sites (RBSs) with degenerate libraries in E. coli

415 (Wang, Isaacs, 2009). Finally, chimeric parts can be constructed by inserting modulating

416 cassettes into native parts. For example, arrays of upstream activation sequences (UASs) were

417 placed in front of a core promoter element to create strong synthetic promoters in S. cerevisiae

418 (Blazeck et al. , 2012) and Yarrowia lipolytica (Blazeck et al. , 2011). If the genetic basis that

419 controls the part performance is relatively clear, modeling can help guide the construction of

420 synthetic parts predicted parameters (Crook and Alper, 2013). For example, a computational

421 model has been established to predict the translational initiation rate of bacterial RBSs, by

422 quantifying the interactions between the $30 \mathrm{~S}$ ribosome complex and the target mRNA molecule

423 (Salis et al. , 2009). Based on a nucleosome architecture model, purely synthetic yeast promoters

424 were obtained with decent strengths (Curran et al. , 2014).

425

4265 High-throughput genotype-phenotype mapping

427 With limited understanding of complex biological systems, "rational" genetic engineering often 428 encounters challenges. Instead, "inverse metabolic engineering” (IME), which isolates mutant 429 strains with a desirable trait first and then proceeds to determine underlying genetic changes, has 430 proved to be a more effective strategy (Bailey et al. , 2002, Santos and Stephanopoulos, 2008). 431 IME not only rapidly improves a target phenotype, but also provides insights to guide future 432 engineering efforts. To fully realize the potential of IME, advanced genotyping and phenotyping 433 techniques are needed to expedite the cycles of creating diversity, selecting best mutants, and 434 mapping relevant genetic changes (Garst et al. , 2013). Genotyping helps to create and track 
435 comprehensive and/or combinatorial diversity across the genome, whereas phenotyping helps to

436 identify mutants with desirable traits in a high-throughput manner.

\subsection{Genotyping}

\subsubsection{Genome-wide libraries}

440 Genome-wide overexpression/knockout libraries are powerful tools to comprehensively

441 investigate the impact of individual genetic modification on a given phenotype (Fig. 2). For

442 overexpression libraries, either genomic fragments (Lynch et al. , 2007) or all the open reading 443 frames (ORFs) under the control of a promoter (Ho et al. , 2009) can be cloned into an

444 extrachromosomal vector. After screen/enrichment, the inserts can be identified through 445 microarray analysis (Lynch et al. , 2004) or DNA sequencing. Genome-wide knockout libraries 446 can be generated by many strategies. Transposon mutagenesis has been optimized for unbiased 447 integration of an antibiotic marker cassette into the entire genome, hence creating a random 448 knockout library (Alexeyev and Shokolenko, 1995, Badarinarayana et al. , 2001). Moreover, all 449 the nonessential genes can be disrupted through homologous recombination, examplified in 450 construction of the yeast deletion collection (Giaever et al. , 2002, Winzeler et al. , 1999) and the 451 Keio E. coli knockout collection (Baba et al. , 2006). In addition to knockout, reduction-of452 function screen has also been applied for genome-wide analysis. Examples include the above453 mentioned screening with asRNAs and RNAi. Knockdown libraries are especially important to 454 study essential genes whose deletion mutations are lethal. For example, insertion of an antibiotic 455 marker into the terminator region to destabilize the mRNA enabled knockdown modification on 456 the essential genes in S. cerevisiae (Breslow et al. , 2008). Notably, there are also technologies 
457 that can create comprehensive genetic libraries including both overexpression and knockdown

458 modifications. For the trackable multiplex recombineering (TRMR), two kinds of synthetic

459 cassettes were designed for promoter replacement: the 'up' cassette containing a strong promoter, 460 and the 'down' cassette containing an inert sequence to replace the native RBS. Through 461 recombineering, these synthetic cassettes were incorporated in front of every gene in E. coli, 462 which led to either increased or decreased expression of a target gene (Warner, Reeder, 2010).

463 Though not demonstrated to create microbial genome-wide libraries yet, CRISPR-mediated 464 knockout, interference and activation can be readily applied for genome-scale analysis as 465 discussed previously.

467 Adding more dimensions to such approaches, i.e. modification at two or even more loci, is 468 necessary because of the non-linear interactions between single genetic variations (Fig. 2). For 469 combinatorial overexpression libraries, the coexpressing genomic libraries (CoGEL) approach 470 was used to construct genomic libraries in a series of vectors (plasmid or fosmid) with 471 compatible replication origins and different resistance markers, which enabled coexistence of 472 two or more genomic inserts in one cell. This approach successfully identified known and novel 473 combinations of genetic changes that conferred improved acid tolerance in E. coli (Nicolaou et 474 al. , 2011). On the other hand, construction of a double-mutant library from single loss-of475 function collections by mating or conjugation has been demonstrated in model organisms such as 476 E. coli (Butland et al. , 2008, Typas et al. , 2008) and S. cerevisiae (Pan et al. , 2004, Tong et al., 477 2001). An impressive application was the depiction of a genome-scale digenic interaction 478 network in S. cerevisiae, by examining 5.4 million gene-gene pairs in a double-mutant library 479 (Costanzo et al. , 2010). However, current protocols to generate genome-wide double-mutant 
480 libraries are quite resource-intensive and time-consuming, as complicated replica-pinning 481 procedures are needed to perform mating, recombination and selection. Therefore, alternative 482 approaches that simplify the introduction of a second mutation on a genome-scale are desirable 483 to speed up the discovery of synergistic modifications. For example, the inherent multiplex 484 capacity of the CRISPR system can be used to create combinatorial genome-wide libraries. As a 485 first step towards this objective, we recently developed a homology integrated CRISPR-Cas (HI486 CRISPR) system for one-step multigene disruptions in $S$. cerevisiae. The mutagenizing 487 homologous recombination donor is integrated at the $5^{\prime}$ of the guide sequence. To increase the 488 gene disruption efficiency, all the HI-CRISPR elements are embedded on a plasmid with ultra489 high copy number. As proof of concept, the simultaneous disruption efficiency of three genes 490 (CAN1, ADE2 and LYP1) ranged from $27 \%$ to $87 \%$, which enabled the identification of desired 491 mutants by random genotyping (Bao, Xiao, 2014).

493 To facilitate subsequent analysis with these genetic libraries, molecular barcodes have been used 494 to monitor the abundance of every mutant strain in a mixed population. Microarray analysis with 495 complementary probes (Pierce et al. , 2006), as well as the "Bar-Seq" method with NGS (Smith 496 et al. , 2009), can be used to quantify the dynamics of barcodes and their linked mutants in 497 various screening experiments, enabling high-throughput mapping of relevant genes to a given 498 phenotype. Such high-throughput capacity explains the wide application of molecular barcodes 499 in analyzing overexpression (Ho, Magtanong, 2009), knockout (Giaever, Chu, 2002) and TRMR 500 libraries (Warner, Reeder, 2010). 


\subsubsection{Genome and transcriptome analysis by next-generation sequencing}

503 Next-generation sequencing (NGS) offers a rapid and inexpensive way to perform genome-scale 504 analysis, whose recent technological advances are reviewed elsewhere (Mardis, 2008, 2013). 505 Here we emphasize on two techniques, comparative genomics (Borneman et al. , 2013) and 506 comparative transcriptomic profiling by RNA-seq (Mutz et al. , 2013, Ozsolak et al. , 2009), 507 which are highly informative in revealing genotype-phenotype relationship, genetic interactions 508 and regulatory networks in microbes.

510 Comparative studies of genome sequences among laboratory strains, industrial strains and 511 natural isolates of the same species can provide insights on the genetic basis of phenotypic 512 differences (Borneman, Pretorius, 2013). For example, the genomes of four wine and two 513 brewing strains of $S$. cerevisiae have been sequenced and analyzed against existing S. cerevisiae 514 genome sequences. The variations between different $S$. cerevisiae strains include single515 nucleotide polymorphisms (SNPs), insertions and deletions, and as novel, strain and allele516 specific ORFs, and genomic rearrangements (Borneman et al. , 2011). Transcriptomic profiling

517 is useful to reveal the impact of different environmental factors on gene expression. For example, 518 transcriptomes of Bacillus subtilis under 104 different environmental and nutritional conditions 519 has been investigated to reveal the structure of transcription network, by grouping 2935 520 promoters into regulons and then linking these regulons with various transcription factors 521 (Nicolas et al. , 2012). For a parental strain and its derivatives obtained from evolutionary 522 engineering or genetic engineering, both genomic and transcriptomic analysis are helpful to 523 understand the genetic basis of acquired traits. Whole genome sequencing (WGS) has been used 524 to track the evolutionary trajectory for aerobic citrate utilization in E. coli (Blount et al. , 2012) 
525 and efficient xylose fermentation in Scheffersomyces stipitis (Smith et al. , 2008). Specifically,

526 mutations that do not affect gene expression can only be identified by genome sequencing. For

527 example, an S. cerevisiae mutant strain with the only lactate transporter gene JEN1 deleted

528 regained the ability to grow on lactate as the sole carbon source during adaptive evolution.

529 Transcriptome analysis provided no clues on the evolved strain; the single-nucleotide changes

530 that conferred an acetate transporter Ady2p with lactate transport activity were only discovered

531 by genome sequencing (de Kok et al. , 2012). On the other hand, transcriptional profiling is

532 important to understand the mechanisms on how genetic changes affect a given trait, especially

533 for those mutations resulting in large-scale perturbation in gene expression. For example,

534 differential expression of hundreds of genes caused by mutations in TFs can only be revealed by

535 transcriptome analysis (Alper, Moxley, 2006).

$537 \quad 5.2$ Reporter-based phenotyping

538 In addition to generating and detecting modifications on a genome-scale, rapid identification of 539 mutants with desired properties from genome-wide libraries is required in IME. In fact, many 540 high-throughput phenotyping methods, including microplate screening (Behrendorff et al. , 541 2013), surface display (Boder and Wittrup, 1997), compartmentalization (Wang et al. , 2014) and 542 fluorescence-activated cell sorting (FACS) (Santoro and Schultz, 2002), have been exploited to 543 detect phenotypes that can be directly screened or selected for (e.g., fluorescence and cell 544 survival, respectively). These methods have been reviewed elsewhere (Leemhuis et al. , 2009) 545 and will not be discussed here. However, phenotyping properties that are not amenable to high546 throughput screening and/or selection (e.g., bulk and fine chemicals production), remains 
547 challenging. To address this limitation, a variety of reporter-based phenotyping methods have

548 been developed to link easily detectable phenotypes to the desired traits.

550 In vivo production of a target molecule can be monitored via a TF-promoter based reporter 551 system. Under the regulation of the molecule-responsive TF and its cognate promoter, 552 expression of the reporter gene results in colorimetric, fluorescent or growth-coupled phenotypes, 553 which are correlated to the concentration of the target molecule and are rapidly identifiable (Fig. 554 3). Using TF-promoter pairs from different microbes, specific activation of transcription factors 555 by dicarboxylic acids and alcohols was coupled to expression of the tetracycline reporter gene. 556 As proof of concept, a biosensor was constructed to identify E. coli mutants with $35 \%$ higher 557 specific productivity of 1-butanol. This biosensor was also incorporated in a synthetic selection 558 method that couples 1-butanol biosynthesis with cell fitness, leading to a 120-fold increase in 1559 butanol production (Dietrich et al., 2013). On the other hand, a feedback-regulated evolution of 560 phenotype (FREP) system was developed to control mutation rates, which are thought to be 561 associated with the probability of finding improved mutants and therefore speed up the adaptive 562 evolution process (Metzgar and Wills, 2000). In the absence of the target molecule, the sensor 563 assembled from the TF-promoter pair activates expression of the actuator and reporter. 564 Expression of the actuator results in an increase in the mutation rate, which may lead to 565 increased production of the target molecule. In response to the increased concentration of the 566 target molecule, the sensor cannot activate the expression of the actuator and reporter, giving rise 567 to a decrease in mutation rate and thus an increase in hereditary stability. Several synthetic $E$. 568 coli TF-promoter pairs were accordingly engineered for better response to the metabolic 569 intermediate isopentenyl pyrophosphate (IPP). Adopting such sensors in FREP was able to 
570 increase tyrosine and isoprenoid production in E. coli (Chou and Keasling, 2013). With a wide

571 dynamic range, high sensitivity and specificity towards a given ligand, the well-characterized

572 ligand-responsive TF and promoter pair always tends to be adopted in an efficient reporter based

573 screening. Yet, promoter characterization is scant in prokaryotes and almost vacant in

574 eukaryotes to date. Also, the most commonly used TF and promoter pairs are unlikely to be

575 responsive to majority of industrially relevant molecules (Dietrich et al. , 2010).

576

577 Due to the lack of effective TF-promoter pairs, synthetic riboswitches responsive to various

578 industrially relevant compounds have become an alternative choice for reporter based screening

579 in eukaryotic organisms. Synthetic riboswitch contains an input domain (encoded in an RNA 580 aptamer) and an output domain (encoded in a ribozyme), which is placed in the 3' untranslated

581 region of a reporter gene. In the absence of a ligand, self-cleavage is catalyzed by the output 582 domain, leading to low gene expression. Binding to a desired molecule by the input domain 583 results in misfolding of the output domain, leading to low cleavage activity and thus high gene 584 expression (Fig. 3). For example, coupled with FACS, an engineered riboswitch was used to 585 identify caffeine demethylase mutants with significantly improved activity and product 586 selectivity (Michener and Smolke, 2012).

\section{Notable examples}

589 Recent advances in genome-scale engineering have significantly enhanced the ability to generate 590 and map multiple functional changes across the entire genome. As such, impressive progress in 591 rewiring genomes to elicit robust, complex traits has been achieved with minimal prior 
592 knowledge of the genetic determinants. A few notable examples of genome-scale engineering 593 will be highlighted in this section.

\subsection{MAGE}

Based on the $\lambda$ Red recombination system, MAGE uses multiple oligos to create combinatorial

597 libraries and optimize gene expression (Fig. 4). Most importantly, the recursive cycles of oligo

598 introduction and allelic replacement are carried out by an automated system, enabling generation

599 of over 4.3 billion genetic variants per day. In one study, the RBSs of 24 genes related to the 1-

600 deoxy-D-xylulose-5-phosphate (DXP) pathway were concurrently modified to increase lycopene

601 production. Mutants with over 5-fold increase in lycopene production were isolated within 3

602 days (after 35 MAGE cycles), representing a significant improvement over previously reported 603 efforts (Wang, Isaacs, 2009). In another study, MAGE was exploited to insert short DNA 604 sequences into the E. Coli choromosome. After 110 MAGE cycles, 18-nt hexa-histidine tag 605 sequences were successfully inserted into 38 essential genes encoding the entire translation 606 machinery, allowing modification and co-purification of large protein complexes and pathways 607 (Wang et al. , 2012).

609 Meanwhile, various MAGE-derived methods were further developed for efficient genome 610 editing. For example, the use of "coselection" MAGE (CoS-MAGE) greatly improves the 611 recombineering efficiency. In the MAGE-generated combinatorial variants, a small portion of 612 cells were observed to harbor multiple mutations (Isaacs, Carr, 2011), which could be selected 613 out in the presence of selective markers. By leveraging co-selection markers around the target 
614 site, oligo-mediated allelic replacement efficiency of over $70 \%$ per viable progeny was achieved

615 (Carr et al. , 2012). Consequently, this approach was used to insert T7 promoter sequences to 12

616 genomic operons related to aromatic amino acid biosynthesis, permitting rapid generation of

617 promoter libraries (Carr, Wang, 2012). A recently-reported microarray oligonucleotide-MAGE

618 (MO-MAGE) method can cost-effectively amplify thousands of oligos from microarray chips.

619 By adopting such technology, T7 promoters were inserted to the upstream of 2585 operons with

620 an average frequency of $0.02 \%$ per locus and 0.4 average insertions per cell (Bonde et al. , 2014).

621 Additionally, the hierarchical conjugative assembly genome engineering (CAGE) method was

622 investigated to reprogram the genetic code. The 32 regions of codon modifications (replacing

623 TAG stop codons with TAA) constructed by MAGE were merged into a single genome through

624 conjugation. Because the TAG stop codon was absent in the resultant strain, this liberated codon

625 can be reassigned to a novel amino acid (Isaacs, Carr, 2011).

626

$627 \quad$ 6.2 TRMR

628 To reduce the unwanted secondary mutations produced by excessive cycles, MAGE is often

629 limited to create modifications to a subset of relevant genes, which requires a prior knowledge of

630 which genes are to be targeted. A complementary method, TRMR, provides a clue to address

631 this limitation. After the introduction of oligos with unique barcodes for recombineering, cells

632 with desired mutations were enriched in a favorable environment and the corresponding genetic

633 modifications could be quantitatively tracked using the barcoded sequences and microarray

634 analysis (Warner, Reeder, 2010) (Fig. 4). As such, thousands of genes that affected E. coli

635 growth in rich, minimal or cellulosic hydrolysate media in the presence of $\beta$-glucoside, D-fucose,

636 valine and methylglyoxal were mapped within one week, permitting identification of large sets 
637 of targets for genome engineering endeavors (Warner, Reeder, 2010). TRMR can also assist

638 MAGE to achieve directed genome engineering via identifying the most relevant genetic

639 modifications. By coupling of TRMR with MAGE, barcoded promoter mutant libraries were

640 first introduced to modify gene expression under different challenging environments. Based on

641 the mapping results, RBS mutant libraries were then designed to retarget genes that dramatically

642 affected the cell growth. Consequently, extensive growth-enhancing mutations were identified 643 from different conditions (Sandoval et al. , 2012).

646 Due to the lack of an efficient recombineering mechanism, it is difficult to apply MAGE or 647 TRMR for genome-scale engineering in eukaryotic microorganisms such as $S$. cerevisiae 648 (DiCarlo et al. , 2013a). On the other hand, RNAi screening has been widely used for functional 649 genomics research in various eukaryotes (Boutros and Ahringer, 2008, Echeverri and Perrimon, 650 2006), yet its applications in MCF engineering are still rare. Recently, a heterologous RNAi 651 pathway was reconstituted in S. cerevisiae (Drinnenberg, Weinberg, 2009), which enables the 652 use of RNAi screening to rapidly understand and engineer complex phenotypes in this yeast (Fig. 653 5). By inserting random genomic DNA fragments into a pair of convergent constitutive 654 promoters, double-stranded RNAs were transcribed in vivo to elicit genome-wide knockdown in 655 the presence of the RNAi pathway ( $\mathrm{Si}$ et al. , 2014). The resultant library was used to 656 successfully identify known suppressors of a telomere-defect mutation $y k u 70 \Delta$ and genetic 657 determinants for improved resistance towards acetic acid and furfural ( $\mathrm{Si}, \mathrm{Luo}, 2014, \mathrm{Xiao}$ and 658 Zhao, 2014). Moreover, compared with the traditional conjugation-based method (Tong, 659 Evangelista, 2001), plasmid-borne RNAi screening is much more convenient in creating 
660 genome-wide perturbations in a modified strain background. Therefore, it is possible to apply a 661 directed evolution strategy on a genome-scale to engineer complex phenotypes in S. cerevisiae

662 (Fig. 5). For example, RNAi-assisted genome evolution (RAGE) was developed to identify 663 three knockdown mutations that acted synergistically to improve acetic acid tolerance 664 substantially in S. cerevisiae (Si, Luo, 2014).

665

6667 Conclusions and perspectives

667 Recent advances in genome-scale engineering have overcome many barriers that constrain strain 668 engineering and therefore greatly expanded our ability to reprogram biological systems. Owing 669 to their high-throughput, complexity, fidelity, and low cost, these new genome-scale engineering 670 technologies have been able to quickly generate vast libraries of combinatorial variations that 671 may have a greater effect on a given phenotype as well as rapidly map the desired trait. 672 Currently these approaches have heretofore been implemented largely in certain model 673 organisms, but adapting them to other industrial microbes is highly desirable. However, this is 674 not a simple task, as the newly developed methods require effective transformation protocols and 675 certain sets of genetic tools, both of which are often absent for industrial fermentation hosts. 676 Whereas establishing genetic manipulation toolbox in less studied organisms will expand the 677 application of genome-scale engineering in industrial settings, high throughput genotyping and 678 phenotyping technologies, such as whole-genome sequencing, transcriptional profiling, and 679 microfluidic and robotic screening, can be combined with classical strain engineering efforts to 680 decrease the length of time for isolation of improved variants and analysis of underlying 681 mechanisms (Crook and Alper, 2012). 
683 As an alternative strategy, rather than dealing with a large number of variants, genome-scale

684 metabolic models can be used to narrow the search space and create functionally rich libraries 685 for optimizing complex traits. For example, employing designs predicted by in silico models has

686 led to not only enhanced production of desired compounds (Choi et al. , 2010, Park et al. , 2007), 687 but also discovery of novel drug targets (Kim et al. , 2011, Lewis et al. , 2010). For a metabolic 688 model, the ability to correctly predict the physiological characteristics of the organism is very 689 important. Hereby, extensive experimental data including high-throughput omics data have been 690 incorporated to validate and improve the quality and the accuracy of a genome-scale metabolic 691 model (Plata et al. , 2010). In such a scenario, the massive data obtained from genome-scale 692 engineering could be significant for construction of more refined and complex metabolic models, 693 which may further benefit the genome-scale engineering in return.

695 Furthermore, laboratory automation may greatly accelerate microbial genome-scale engineering. 696 By eliminating human intervention, laboratory automation promises to improve productivity and 697 reliability, increase throughput, and reduce experimental error rates due to human factors 698 (Linshiz et al. , 2013). Pharmaceutical industry has heavily relied on automation technologies to 699 identify new drug lead compounds by screening small-molecule libraries (Nettekoven and 700 Thomas, 2002). Automation platforms have also been developed for bottom-up construction of 701 genetic circuits and metabolic pathways from modular parts (Densmore and Hassoun, 2012, 702 Dharmadi et al. , 2014), integrating software for assembly algorithm and data management, as 703 well as hardware for liquid handling and DNA construct analysis. For biological system 704 engineering, the promise of automation has been demonstrated by several recent examples 
705 (Esvelt et al. , 2011, Wang, Isaacs, 2009). Phage-assisted continuous evolution (PACE) 706 executed 200 rounds of protein evolution in 8 days, during which targeted activities effectively 707 emerged from undetectable levels (Esvelt, Carlson, 2011). Moreover, MAGE created over 4.3 708 billion combinatorial variants per day, enabling 5-fold increase in lycopene production in 3 days 709 (Wang, Isaacs, 2009). Future development of automation-friendly protocols, including both 710 strain variant generation and phenotypic screening methods, may help to increase the use of 711 laboratory automation in genome-scale engineering of microbial cell factories. Finally, we 712 envision that, with endeavors on but not limited to the above mentioned aspects, genome-scale 713 engineering can accomplish intensive reprogramming of microbial metabolism for multiple 714 engineering purposes.

\section{Conflict of interest statement}

717 The authors declare that there are no conflicts of interest.

\section{Acknowledgments}

720 We gratefully acknowledge financial support from National Institutes of Health (GM077596),

721 Defense Advanced Research Projects Agency (DARPA), Department of Energy (DE722 SC0008743), and National Academies Keck Futures Initiative on genome engineering. 


\section{References}

Albert H, Dale EC, Lee E, Ow DW. Site-specific integration of DNA into wild-type and mutant lox sites placed in the plant genome. Plant J. 1995;7:649-59.

Alexeyev MF, Shokolenko IN. Mini-Tn10 transposon derivatives for insertion mutagenesis and gene delivery into the chromosome of gram-negative bacteria. Gene. 1995;160:59-62.

Alper H, Fischer C, Nevoigt E, Stephanopoulos G. Tuning genetic control through promoter engineering. Proc Natl Acad Sci U S A. 2005;102:12678-83.

Alper H, Moxley J, Nevoigt E, Fink GR, Stephanopoulos G. Engineering yeast transcription machinery for improved ethanol tolerance and production. Science. 2006;314:1565-8.

Alper H, Stephanopoulos G. Global transcription machinery engineering: a new approach for improving cellular phenotype. Metab Eng. 2007;9:258-67.

Annaluru N, Muller H, Mitchell LA, Ramalingam S, Stracquadanio G, Richardson SM, et al. Total synthesis of a functional designer eukaryotic chromosome. Science. 2014;344:55-8.

Aouida M, Piatek MJ, Bangarusamy DK, Mahfouz MM. Activities and specificities of homodimeric TALENs in Saccharomyces cerevisiae. Curr Genet. 2014;60:61-74.

Baba T, Ara T, Hasegawa M, Takai Y, Okumura Y, Baba M, et al. Construction of Escherichia coli K-12 in-frame, single-gene knockout mutants: the Keio collection. Mol Syst Biol. 2006;2:2006.0008.

Badarinarayana V, Estep PW, 3rd, Shendure J, Edwards J, Tavazoie S, Lam F, et al. Selection analyses of insertional mutants using subgenic-resolution arrays. Nat Biotechnol. 2001;19:1060-5.

Bailey JE, Sburlati A, Hatzimanikatis V, Lee K, Renner WA, Tsai PS. Inverse metabolic engineering: a strategy for directed genetic engineering of useful phenotypes. Biotechnol Bioeng. 2002;79:568-79.

Bao Z, Xiao H, Liang J, Zhang L, Xiong X, Sun N, et al. Homology-Integrated CRISPR-Cas (HICRISPR) system for one-step multigene disruption in Saccharomyces cerevisiae. ACS Synth Biol. 2014.

Behrendorff JB, Vickers CE, Chrysanthopoulos P, Nielsen LK. 2,2-Diphenyl-1-picrylhydrazyl as a screening tool for recombinant monoterpene biosynthesis. Microb Cell Fact. 2013;12:76.

Bibikova M, Carroll D, Segal DJ, Trautman JK, Smith J, Kim YG, et al. Stimulation of homologous recombination through targeted cleavage by chimeric nucleases. Mol Cell Biol. 2001;21:289-97.

Bikard D, Jiang W, Samai P, Hochschild A, Zhang F, Marraffini LA. Programmable repression and activation of bacterial gene expression using an engineered CRISPR-Cas system. Nucleic Acids Res. 2013;41:7429-37.

Biot-Pelletier D, Martin VJJ. Evolutionary engineering by genome shuffling. Appl Microbiol Biotechnol. 2014;98:3877-87. 
Blazeck J, Garg R, Reed B, Alper HS. Controlling promoter strength and regulation in Saccharomyces cerevisiae using synthetic hybrid promoters. Biotechnol Bioeng. 2012;109:2884-95.

Blazeck J, Liu L, Redden H, Alper H. Tuning gene expression in Yarrowia lipolytica by a hybrid promoter approach. Appl Environ Microbiol. 2011;77:7905-14.

Blount ZD, Barrick JE, Davidson CJ, Lenski RE. Genomic analysis of a key innovation in an experimental Escherichia coli population. Nature. 2012;489:513-8.

Boder ET, Wittrup KD. Yeast surface display for screening combinatorial polypeptide libraries. Nat Biotechnol. 1997;15:553-7.

Bonde MT, Kosuri S, Genee HJ, Sarup-Lytzen K, Church GM, Sommer MO, et al. Direct mutagenesis of thousands of genomic targets using microarray-derived oligonucleotides. ACS Synth Biol. 2014.

Borneman AR, Desany BA, Riches D, Affourtit JP, Forgan AH, Pretorius IS, et al. Whole-genome comparison reveals novel genetic elements that characterize the genome of industrial strains of Saccharomyces cerevisiae. PLoS Genet. 2011;7:e1001287.

Borneman AR, Pretorius IS, Chambers PJ. Comparative genomics: a revolutionary tool for wine yeast strain development. Curr Opin Biotechnol. 2013;24:192-9.

Boutros M, Ahringer J. The art and design of genetic screens: RNA interference. Nat Rev Genet. 2008;9:554-66.

Boyle NR, Reynolds TS, Evans R, Lynch M, Gill RT. Recombineering to homogeneity: extension of multiplex recombineering to large-scale genome editing. Biotechnol J. 2013;8:515-22.

Breslow DK, Cameron DM, Collins SR, Schuldiner M, Stewart-Ornstein J, Newman HW, et al. A comprehensive strategy enabling high-resolution functional analysis of the yeast genome. Nat Methods. 2008;5:711-8.

Briggs AW, Rios X, Chari R, Yang L, Zhang F, Mali P, et al. Iterative capped assembly: rapid and scalable synthesis of repeat-module DNA such as TAL effectors from individual monomers. Nucleic Acids Res. 2012;40:e117.

Buchholz F, Stewart AF. Alteration of Cre recombinase site specificity by substrate-linked protein evolution. Nat Biotechnol. 2001;19:1047-52.

Butland G, Babu M, Diaz-Mejia JJ, Bohdana F, Phanse S, Gold B, et al. eSGA: E. coli synthetic genetic array analysis. Nat Methods. 2008;5:789-95.

Carbery ID, Ji D, Harrington A, Brown V, Weinstein EJ, Liaw L, et al. Targeted genome modification in mice using zinc-finger nucleases. Genetics. 2010;186:451-9.

Carr PA, Church GM. Genome engineering. Nat Biotechnol. 2009;27:1151-62.

Carr PA, Wang HH, Sterling B, Isaacs FJ, Lajoie MJ, Xu G, et al. Enhanced multiplex genome engineering through co-operative oligonucleotide co-selection. Nucleic Acids Res. 2012;40:e132. 
Carroll D. Genome engineering with zinc-finger nucleases. Genetics. 2011;188:773-82.

Cello J, Paul AV, Wimmer E. Chemical synthesis of poliovirus cDNA: generation of infectious virus in the absence of natural template. Science. 2002;297:1016-8.

Chan LY, Kosuri S, Endy D. Refactoring bacteriophage T7. Mol Syst Biol. 2005;1:2005 0018.

Chao R, Yuan Y, Zhao H. Recent advances in DNA assembly technologies. FEMS Yeast Res. 2014.

Cho SW, Kim S, Kim Y, Kweon J, Kim HS, Bae S, et al. Analysis of off-target effects of CRISPR/Casderived RNA-guided endonucleases and nickases. Genome Res. 2014;24:132-41.

Choi HS, Lee SY, Kim TY, Woo HM. In silico identification of gene amplification targets for improvement of lycopene production. Appl Environ Microbiol. 2010;76:3097-105.

Chou HH, Keasling JD. Programming adaptive control to evolve increased metabolite production. Nat Commun. 2013;4:2595.

Cong L, Ran FA, Cox D, Lin SL, Barretto R, Habib N, et al. Multiplex genome engineering using CRISPR/Cas systems. Science. 2013;339:819-23.

Costanzo M, Baryshnikova A, Bellay J, Kim Y, Spear ED, Sevier CS, et al. The genetic landscape of a cell. Science. 2010;327:425-31.

Cox MM, Layton SL, Jiang T, Cole K, Hargis BM, Berghman LR, et al. Scarless and site-directed mutagenesis in Salmonella enteritidis chromosome. BMC Biotechnol. 2007;7:59.

Crook N, Alper HS. Classical Strain Improvement. Engineering complex phenotypes in industrial strains: John Wiley \& Sons, Inc.; 2012. p. 1-33.

Crook N, Alper HS. Model-based design of synthetic, biological systems. Chem Eng Sci. 2013;103:2-11.

Curran KA, Crook NC, Karim AS, Gupta A, Wagman AM, Alper HS. Design of synthetic yeast promoters via tuning of nucleosome architecture. Nat Commun. 2014;5.

Datta S, Costantino N, Zhou X, Court DL. Identification and analysis of recombineering functions from Gram-negative and Gram-positive bacteria and their phages. Proc Natl Acad Sci U S A. 2008;105:1626-31.

de Kok S, Nijkamp JF, Oud B, Roque FC, de Ridder D, Daran JM, et al. Laboratory evolution of new lactate transporter genes in a jen1Delta mutant of Saccharomyces cerevisiae and their identification as ADY2 alleles by whole-genome resequencing and transcriptome analysis. FEMS Yeast Res. 2012.

Deiters A, Cropp TA, Mukherji M, Chin JW, Anderson JC, Schultz PG. Adding amino acids with novel reactivity to the genetic code of Saccharomyces cerevisiae. J Am Chem Soc. 2003;125:11782-3.

Deltcheva E, Chylinski K, Sharma CM, Gonzales K, Chao Y, Pirzada ZA, et al. CRISPR RNA maturation by trans-encoded small RNA and host factor RNase III. Nature. 2011;471:602-7. 
Densmore D, Hassoun S. Design automation for synthetic biological systems. Design \& Test of Computers, IEEE. 2012;29:7-20.

Dharmadi Y, Patel K, Shapland E, Hollis D, Slaby T, Klinkner N, et al. High-throughput, cost-effective verification of structural DNA assembly. Nucleic Acids Res. 2014;42:e22.

DiCarlo JE, Conley AJ, Penttila M, Jantti J, Wang HH, Church GM. Yeast oligo-mediated genome engineering (YOGE). ACS Synth Biol. 2013a;2:741-9.

Dicarlo JE, Norville JE, Mali P, Rios X, Aach J, Church GM. Genome engineering in Saccharomyces cerevisiae using CRISPR-Cas systems. Nucleic Acids Res. 2013b;41:4336-43.

Dietrich JA, McKee AE, Keasling JD. High-throughput metabolic engineering: advances in smallmolecule screening and selection. Annu Rev Biochem. 2010;79:563-90.

Dietrich JA, Shis DL, Alikhani A, Keasling JD. Transcription factor-based screens and synthetic selections for microbial small-molecule biosynthesis. ACS Synth Biol. 2013;2:47-58.

Drinnenberg IA, Weinberg DE, Xie KT, Mower JP, Wolfe KH, Fink GR, et al. RNAi in budding yeast. Science. 2009;326:544-50.

Dymecki SM. Flp recombinase promotes site-specific DNA recombination in embryonic stem cells and transgenic mice. Proc Natl Acad Sci U S A. 1996;93:6191-6.

Dymond JS, Richardson SM, Coombes CE, Babatz T, Muller H, Annaluru N, et al. Synthetic chromosome arms function in yeast and generate phenotypic diversity by design. Nature. 2011;477:471-6.

Echeverri CJ, Perrimon N. High-throughput RNAi screening in cultured cells: a user's guide. Nat Rev Genet. 2006;7:373-84.

Eckert SE, Dziva F, Chaudhuri RR, Langridge GC, Turner DJ, Pickard DJ, et al. Retrospective application of transposon-directed insertion site sequencing to a library of signature-tagged miniTn5Km2 mutants of Escherichia coli O157:H7 screened in cattle. J Bacteriol. 2011;193:1771-6.

Ellis HM, Yu D, DiTizio T, Court DL. High efficiency mutagenesis, repair, and engineering of chromosomal DNA using single-stranded oligonucleotides. Proc Natl Acad Sci U S A. 2001;98:6742-6.

Enyeart PJ, Chirieleison SM, Dao MN, Perutka J, Quandt EM, Yao J, et al. Generalized bacterial genome editing using mobile group II introns and Cre-lox. Mol Syst Biol. 2013;9:685.

Esvelt KM, Carlson JC, Liu DR. A system for the continuous directed evolution of biomolecules. Nature. 2011;472:499-503.

Esvelt KM, Wang HH. Genome-scale engineering for systems and synthetic biology. Mol Syst Biol. 2013;9:17. 
Farzadfard F, Perli SD, Lu TK. Tunable and multifunctional eukaryotic transcription factors based on CRISPR/Cas. ACS Synth Biol. 2013;2:604-13.

Feng Z, Zhang B, Ding W, Liu X, Yang DL, Wei P, et al. Efficient genome editing in plants using a CRISPR/Cas system. Cell Res. 2013;23:1229-32.

Fire A, Xu SQ, Montgomery MK, Kostas SA, Driver SE, Mello CC. Potent and specific genetic interference by double-stranded RNA in Caenorhabditis elegans. Nature. 1998;391:806-11.

Forsyth RA, Haselbeck RJ, Ohlsen KL, Yamamoto RT, Xu H, Trawick JD, et al. A genome-wide strategy for the identification of essential genes in Staphylococcus aureus. Mol Microbiol. 2002;43:1387400 .

Fu J, Bian X, Hu S, Wang H, Huang F, Seibert PM, et al. Full-length RecE enhances linear-linear homologous recombination and facilitates direct cloning for bioprospecting. Nat Biotechnol. 2012;30:440-6.

Fu YF, Foden JA, Khayter C, Maeder ML, Reyon D, Joung JK, et al. High-frequency off-target mutagenesis induced by CRISPR-Cas nucleases in human cells. Nat Biotechnol. 2013;31:822-6.

Fu YF, Sander JD, Reyon D, Cascio VM, Joung JK. Improving CRISPR-Cas nuclease specificity using truncated guide RNAs. Nat Biotechnol. 2014;32:279-84.

Garst A, Lynch M, Evans R, Gill RT. Strategies for the multiplex mapping of genes to traits. Microb Cell Fact. 2013;12:99.

Giaever G, Chu AM, Ni L, Connelly C, Riles L, Veronneau S, et al. Functional profiling of the Saccharomyces cerevisiae genome. Nature. 2002;418:387-91.

Gibson DG, Benders GA, Andrews-Pfannkoch C, Denisova EA, Baden-Tillson H, Zaveri J, et al. Complete chemical synthesis, assembly, and cloning of a Mycoplasma genitalium genome. Science. 2008;319:1215-20.

Gibson DG, Glass JI, Lartigue C, Noskov VN, Chuang RY, Algire MA, et al. Creation of a bacterial cell controlled by a chemically synthesized genome. Science. 2010;329:52-6.

Gilbert LA, Larson MH, Morsut L, Liu Z, Brar GA, Torres SE, et al. CRISPR-mediated modular RNAguided regulation of transcription in eukaryotes. Cell. 2013;154:442-51.

Gordley RM, Gersbach CA, Barbas CF, 3rd. Synthesis of programmable integrases. Proc Natl Acad Sci U S A. 2009;106:5053-8.

Hamer L, DeZwaan TM, Montenegro-Chamorro MV, Frank SA, Hamer JE. Recent advances in largescale transposon mutagenesis. Curr Opin Chem Biol. 2001;5:67-73.

Hannon GJ. RNA interference. Nature. 2002;418:244-51. 
Ho CH, Magtanong L, Barker SL, Gresham D, Nishimura S, Natarajan P, et al. A molecular barcoded yeast ORF library enables mode-of-action analysis of bioactive compounds. Nat Biotechnol. 2009;27:369-77.

Horvath P, Romero DA, Coute-Monvoisin AC, Richards M, Deveau H, Moineau S, et al. Diversity, activity, and evolution of CRISPR loci in Streptococcus thermophilus. J Bacteriol. 2008;190:140112.

Hutchison CA, Peterson SN, Gill SR, Cline RT, White O, Fraser CM, et al. Global transposon mutagenesis and a minimal Mycoplasma genome. Science. 1999;286:2165-9.

Hwang WY, Fu YF, Reyon D, Maeder ML, Tsai SQ, Sander JD, et al. Efficient genome editing in Zebrafish using a CRISPR-Cas system. Nat Biotechnol. 2013;31:227-9.

Iglehart JD, Silver DP. Synthetic lethality--a new direction in cancer-drug development. N Engl J Med. 2009;361:189-91.

Isaacs FJ, Carr PA, Wang HH, Lajoie MJ, Sterling B, Kraal L, et al. Precise manipulation of chromosomes in vivo enables genome-wide codon replacement. Science. 2011;333:348-53.

Jeong J, Cho N, Jung D, Bang D. Genome-scale genetic engineering in Escherichia coli. Biotechnol Adv. 2013;31:804-10.

Jiang W, Bikard D, Cox D, Zhang F, Marraffini LA. RNA-guided editing of bacterial genomes using CRISPR-Cas systems. Nat Biotechnol. 2013;31:233-9.

Kang Z, Zhang CZ, Zhang JL, Jin P, Zhang J, Du GC, et al. Small RNA regulators in bacteria: powerful tools for metabolic engineering and synthetic biology. Appl Microbiol Biotechnol. 2014;98:341324.

Karas BJ, Tagwerker C, Yonemoto IT, Hutchison CA, 3rd, Smith HO. Cloning the Acholeplasma laidlawii PG-8A genome in Saccharomyces cerevisiae as a yeast centromeric plasmid. ACS Synth Biol. 2012;1:22-8.

Karberg M, Guo H, Zhong J, Coon R, Perutka J, Lambowitz AM. Group II introns as controllable gene targeting vectors for genetic manipulation of bacteria. Nat Biotechnol. 2001;19:1162-7.

Karow M, Calos MP. The therapeutic potential of PhiC31 integrase as a gene therapy system. Expert Opin Biol Ther. 2011;11:1287-96.

Keasling JD. Manufacturing molecules through metabolic engineering. Science. 2010;330:1355-8.

Kim E, Kim S, Kim DH, Choi BS, Choi IY, Kim JS. Precision genome engineering with programmable DNA-nicking enzymes. Genome Res. 2012;22:1327-33.

Kim HU, Kim SY, Jeong H, Kim TY, Kim JJ, Choy HE, et al. Integrative genome-scale metabolic analysis of Vibrio vulnificus for drug targeting and discovery. Mol Syst Biol. 2011;7:460. 
Klein-Marcuschamer D, Stephanopoulos G. Assessing the potential of mutational strategies to elicit new phenotypes in industrial strains. Proc Natl Acad S U S A. 2008;105:2319-24.

Lajoie MJ, Gregg CJ, Mosberg JA, Washington GC, Church GM. Manipulating replisome dynamics to enhance lambda Red-mediated multiplex genome engineering. Nucleic Acids Res. 2012;40:e170.

Lee JW, Na D, Park JM, Lee J, Choi S, Lee SY. Systems metabolic engineering of microorganisms for natural and non-natural chemicals. Nat Chem Biol. 2012;8:536-46.

Lee JY, Yang KS, Jang SA, Sung BH, Kim SC. Engineering butanol-tolerance in Escherichia coli with artificial transcription factor libraries. Biotechnol Bioeng. 2011;108:742-9.

Leemhuis H, Kelly RM, Dijkhuizen L. Directed evolution of enzymes: library screening strategies. IUBMB Life. 2009;61:222-8.

Lewis NE, Schramm G, Bordbar A, Schellenberger J, Andersen MP, Cheng JK, et al. Large-scale in silico modeling of metabolic interactions between cell types in the human brain. Nat Biotechnol. 2010;28:1279-85.

Li L, Wu LP, Chandrasegaran S. Functional domains in Fok I restriction endonuclease. Proc Natl Acad Sci U S A. 1992;89:4275-9.

Li T, Huang S, Zhao X, Wright DA, Carpenter S, Spalding MH, et al. Modularly assembled designer TAL effector nucleases for targeted gene knockout and gene replacement in eukaryotes. Nucleic Acids Res. 2011;39:6315-25.

Liang J, Chao R, Abil Z, Bao Z, Zhao H. Fairytale: a high-throughput TAL effector synthesis platform. ACS Synth Biol. 2014;3:67-73.

Lin Z, Zhang Y, Wang J. Engineering of transcriptional regulators enhances microbial stress tolerance. Biotechnol Adv. 2013;31:986-91.

Linshiz G, Stawski N, Poust S, Bi C, Keasling JD, Hillson NJ. PaR-PaR laboratory automation platform. ACS Synth Biol. 2013;2:216-22.

Lopez-Maury L, Marguerat S, Bahler J. Tuning gene expression to changing environments: from rapid responses to evolutionary adaptation. Nat Rev Genet. 2008;9:583-93.

Lynch MD, Gill RT, Stephanopoulos G. Mapping phenotypic landscapes using DNA micro-arrays. Metab Eng. 2004;6:177-85.

Lynch MD, Warnecke T, Gill RT. SCALEs: multiscale analysis of library enrichment. Nat Methods. 2007;4:87-93.

Maggert KA, Gong WJ, Golic KG. Methods for homologous recombination in Drosophila. Methods Mol Biol. 2008;420:155-74.

Mali P, Yang L, Esvelt KM, Aach J, Guell M, DiCarlo JE, et al. RNA-guided human genome engineering via Cas9. Science. 2013;339:823-6. 
Malyshev DA, Dhami K, Lavergne T, Chen T, Dai N, Foster JM, et al. A semi-synthetic organism with an expanded genetic alphabet. Nature. 2014;509:385-8.

Mardis ER. Next-generation DNA sequencing methods. Annu Rev Genomics Hum Genet. 2008;9:387402.

Mardis ER. Next-generation sequencing platforms. Annu Rev Anal Chem (Palo Alto Calif). 2013;6:287303.

Matzas M, Stahler PF, Kefer N, Siebelt N, Boisguerin V, Leonard JT, et al. High-fidelity gene synthesis by retrieval of sequence-verified DNA identified using high-throughput pyrosequencing. Nat Biotechnol. 2010;28:1291-4.

Meng J, Kanzaki G, Meas D, Lam CK, Crummer H, Tain J, et al. A genome-wide inducible phenotypic screen identifies antisense RNA constructs silencing Escherichia coli essential genes. FEMS Microbiol Lett. 2012;329:45-53.

Meng X, Noyes MB, Zhu LJ, Lawson ND, Wolfe SA. Targeted gene inactivation in Zebrafish using engineered zinc-finger nucleases. Nat Biotechnol. 2008;26:695-701.

Metzgar D, Wills C. Evidence for the adaptive evolution of mutation rates. Cell. 2000;101:581-4.

Michel F, Ferat JL. Structure and activities of group II introns. Annu Rev Biochem. 1995;64:435-61.

Michener JK, Smolke CD. High-throughput enzyme evolution in Saccharomyces cerevisiae using a synthetic RNA switch. Metab Eng. 2012;14:306-16.

Mizuuchi M, Mizuuchi K. Integrative recombination of bacteriophage lambda: extent of the DNA sequence involved in attachment site function. Proc Natl Acad Sci U S A. 1980;77:3220-4.

Moure CM, Gimble FS, Quiocho FA. Crystal structures of I-SceI complexed to nicked DNA substrates: snapshots of intermediates along the DNA cleavage reaction pathway. Nucleic Acids Res. 2008;36:3287-96.

Mueller S, Coleman JR, Wimmer E. Putting synthesis into biology: a viral view of genetic engineering through de novo gene and genome synthesis. Chem Biol. 2009;16:337-47.

Murphy KC. Use of bacteriophage lambda recombination functions to promote gene replacement in Escherichia coli. J Bacteriol. 1998;180:2063-71.

Mussolino C, Morbitzer R, Lutge F, Dannemann N, Lahaye T, Cathomen T. A novel TALE nuclease scaffold enables high genome editing activity in combination with low toxicity. Nucleic Acids Res. 2011;39:9283-93.

Mutz KO, Heilkenbrinker A, Lonne M, Walter JG, Stahl F. Transcriptome analysis using next-generation sequencing. Curr Opin Biotechnol. 2013;24:22-30.

Na D, Yoo SM, Chung H, Park H, Park JH, Lee SY. Metabolic engineering of Escherichia coli using synthetic small regulatory RNAs. Nat Biotechnol. 2013;31:170-4. 
Nagy A. Cre recombinase: the universal reagent for genome tailoring. Genesis. 2000;26:99-109.

Nakashima N, Tamura T, Good L. Paired termini stabilize antisense RNAs and enhance conditional gene silencing in Escherichia coli. Nucleic Acids Res. 2006;34:e138.

Nettekoven M, Thomas AW. Accelerating drug discovery by integrative implementation of laboratory automation in the work flow. Curr Med Chem. 2002;9:2179-90.

Neumann H, Wang K, Davis L, Garcia-Alai M, Chin JW. Encoding multiple unnatural amino acids via evolution of a quadruplet-decoding ribosome. Nature. 2010;464:441-4.

Nevoigt E, Kohnke J, Fischer CR, Alper H, Stahl U, Stephanopoulos G. Engineering of promoter replacement cassettes for fine-tuning of gene expression in Saccharomyces cerevisiae. Appl Environ Microbiol. 2006;72:5266-73.

Nicolaou SA, Gaida SM, Papoutsakis ET. Coexisting/Coexpressing Genomic Libraries (CoGeL) identify interactions among distantly located genetic loci for developing complex microbial phenotypes. Nucleic Acids Res. 2011;39:e152.

Nicolas P, Mader U, Dervyn E, Rochat T, Leduc A, Pigeonneau N, et al. Condition-dependent transcriptome reveals high-level regulatory architecture in Bacillus subtilis. Science. 2012;335:1103-6.

Ochiai H, Fujita K, Suzuki K, Nishikawa M, Shibata T, Sakamoto N, et al. Targeted mutagenesis in the sea urchin embryo using zinc-finger nucleases. Genes Cells. 2010;15:875-85.

Ozsolak F, Platt AR, Jones DR, Reifenberger JG, Sass LE, McInerney P, et al. Direct RNA sequencing. Nature. 2009;461:814-8.

Pan X, Yuan DS, Xiang D, Wang X, Sookhai-Mahadeo S, Bader JS, et al. A robust toolkit for functional profiling of the yeast genome. Mol Cell. 2004;16:487-96.

Park JH, Lee KH, Kim TY, Lee SY. Metabolic engineering of Escherichia coli for the production of Lvaline based on transcriptome analysis and in silico gene knockout simulation. Proc Natl Acad Sci U S A. 2007;104:7797-802.

Park K-S, Lee D-k, Lee H, Lee Y, Jang Y-S, Kim YH, et al. Phenotypic alteration of eukaryotic cells using randomized libraries of artificial transcription factors. Nat Biotechnol. 2003;21:1208-14.

Park KS, Jang YS, Lee H, Kim JS. Phenotypic alteration and target gene identification using combinatorial libraries of zinc finger proteins in prokaryotic cells. J Bacteriol. 2005; 187:5496-9.

Pavletich NP, Pabo CO. Zinc finger-DNA recognition: crystal structure of a Zif268-DNA complex at 2.1 A. Science. 1991;252:809-17.

Perutka J, Wang W, Goerlitz D, Lambowitz AM. Use of computer-designed group II introns to disrupt Escherichia coli DExH/D-box protein and DNA helicase genes. J Mol Biol. 2004;336:421-39. 
1022

1023

1024

1025

1026

1027

1028

1029

1030

1031

1032

1033

1034

1035

1036

1037

1038

1039

1040

1041

1042

1043

1044

1045

1046

1047

1048

1049

1050

1051

1052

1053

1054

1055

Pfleger BF, Pitera DJ, Smolke CD, Keasling JD. Combinatorial engineering of intergenic regions in operons tunes expression of multiple genes. Nat Biotechnol. 2006;24:1027-32.

Pierce SE, Fung EL, Jaramillo DF, Chu AM, Davis RW, Nislow C, et al. A unique and universal molecular barcode array. Nat Methods. 2006;3:601-3.

Plata G, Hsiao TL, Olszewski KL, Llinas M, Vitkup D. Reconstruction and flux-balance analysis of the Plasmodium falciparum metabolic network. Mol Syst Biol. 2010;6:408.

Portnoy VA, Bezdan D, Zengler K. Adaptive laboratory evolution - harnessing the power of biology for metabolic engineering. Curr Opin Biotechnol. 2011;22:590-4.

Qi LS, Arkin AP. A versatile framework for microbial engineering using synthetic non-coding RNAs. Nat Rev Microbiol. 2014;12:341-54.

Qi LS, Larson MH, Gilbert LA, Doudna JA, Weissman JS, Arkin AP, et al. Repurposing CRISPR as an RNA-guided platform for sequence-specific control of gene expression. Cell. 2013;152:1173-83.

Quan JY, Saaem I, Tang N, Ma SM, Negre N, Gong H, et al. Parallel on-chip gene synthesis and application to optimization of protein expression. Nat Biotechnol. 2011;29:449-+.

Rabinovitch-Deere CA, Oliver JWK, Rodriguez GM, Atsumi S. Synthetic biology and metabolic engineering approaches to produce biofuels. Chem Rev. 2013;113:4611-32.

Ramirez CL, Foley JE, Wright DA, Muller-Lerch F, Rahman SH, Cornu TI, et al. Unexpected failure rates for modular assembly of engineered zinc fingers. Nat Methods. 2008;5:374-5.

Ryu Y, Schultz PG. Efficient incorporation of unnatural amino acids into proteins in Escherichia coli. Nat Methods. 2006;3:263-5.

Salis HM, Mirsky EA, Voigt CA. Automated design of synthetic ribosome binding sites to control protein expression. Nat Biotechnol. 2009;27:946-50.

Sandoval NR, Kim JY, Glebes TY, Reeder PJ, Aucoin HR, Warner JR, et al. Strategy for directing combinatorial genome engineering in Escherichia coli. Proc Natl Acad Sci U S A. 2012;109:105405 .

Santoro SW, Schultz PG. Directed evolution of the site specificity of Cre recombinase. Proc Natl Acad Sci U S A. 2002;99:4185-90.

Santos CN, Stephanopoulos G. Combinatorial engineering of microbes for optimizing cellular phenotype. Curr Opin Chem Biol. 2008;12:168-76.

Sawitzke JA, Costantino N, Li XT, Thomason LC, Bubunenko M, Court C, et al. Probing cellular processes with oligo-mediated recombination and using the knowledge gained to optimize recombineering. J Mol Biol. 2011;407:45-59.

Sawitzke JA, Thomason LC, Costantino N, Bubunenko M, Datta S, Court DL. Recombineering: in vivo genetic engineering in E. coli, S. enterica, and beyond. Methods Enzymol. 2007;421:171-99. 
Schlake T, Bode J. Use of mutated FLP recognition target (FRT) sites for the exchange of expression cassettes at defined chromosomal loci. Biochemistry. 1994;33:12746-51.

Segal DJ, Meckler JF. Genome engineering at the dawn of the golden age. Annu Rev Genomics Hum Genet. 2013;14:135-58.

Shao L, Hu S, Yang Y, Gu Y, Chen J, Jiang W, et al. Targeted gene disruption by use of a group II intron (targetron) vector in Clostridium acetobutylicum. Cell Res. 2007;17:963-5.

Shao Z, Rao G, Li C, Abil Z, Luo Y, Zhao H. Refactoring the silent spectinabilin gene cluster using a plug-and-play scaffold. ACS Synth Biol. 2013;2:662-9.

Sharan SK, Thomason LC, Kuznetsov SG, Court DL. Recombineering: a homologous recombinationbased method of genetic engineering. Nat Protoc. 2009;4:206-23.

Shen B, Zhang W, Zhang J, Zhou J, Wang J, Chen L, et al. Efficient genome modification by CRISPRCas9 nickase with minimal off-target effects. Nat Methods. 2014;11:399-402.

Si T, Luo Y, Bao Z, Zhao H. RNAi-assisted genome evolution in Saccharomyces cerevisiae for complex phenotype engineering. ACS Synth Biol. 2014.

Silva G, Poirot L, Galetto R, Smith J, Montoya G, Duchateau P, et al. Meganucleases and other tools for targeted genome engineering: perspectives and challenges for gene therapy. Curr Gene Ther. 2011;11:11-27.

Smith AM, Heisler LE, Mellor J, Kaper F, Thompson MJ, Chee M, et al. Quantitative phenotyping via deep barcode sequencing. Genome Res. 2009;19:1836-42.

Smith DR, Quinlan AR, Peckham HE, Makowsky K, Tao W, Woolf B, et al. Rapid whole-genome mutational profiling using next-generation sequencing technologies. Genome Res. 2008;18:1638-42.

Sternberg N, Hamilton D, Hoess R. Bacteriophage-p1 site-specific recombination .2. Recombination between loxp and the bacterial chromosome. J Mol Biol. 1981;150:487-507.

Sun J, Shao Z, Zhao H, Nair N, Wen F, Xu JH, et al. Cloning and characterization of a panel of constitutive promoters for applications in pathway engineering in Saccharomyces cerevisiae. Biotechnol Bioeng. 2012a;109:2082-92.

Sun N, Abil Z, Zhao H. Recent advances in targeted genome engineering in mammalian systems. Biotechnol J. 2012b;7:1074-87.

Sun N, Liang J, Abil Z, Zhao H. Optimized TAL effector nucleases (TALENs) for use in treatment of sickle cell disease. Mol Biosyst. 2012c;8:1255-63.

Swingle B, Markel E, Costantino N, Bubunenko MG, Cartinhour S, Court DL. Oligonucleotide recombination in Gram-negative bacteria. Mol Microbiol. 2010;75:138-48.

Tang N, Ma SY, Tian JD. New tools for cost-effective DNA synthesis. Synthetic Biology: Tools and Applications 2013. p. 3-21. 
Tong AH, Evangelista M, Parsons AB, Xu H, Bader GD, Page N, et al. Systematic genetic analysis with ordered arrays of yeast deletion mutants. Science. 2001;294:2364-8.

Townsend JA, Wright DA, Winfrey RJ, Fu F, Maeder ML, Joung JK, et al. High-frequency modification of plant genes using engineered zinc-finger nucleases. Nature. 2009;459:442-5.

Turan S, Bode J. Site-specific recombinases: from tag-and-target- to tag-and-exchange-based genomic modifications. FASEB J. 2011;25:4088-107.

Turan S, Galla M, Ernst E, Qiao J, Voelkel C, Schiedlmeier B, et al. Recombinase-mediated cassette exchange (RMCE): traditional concepts and current challenges. J Mol Biol. 2011;407:193-221.

Turan S, Kuehle J, Schambach A, Baum C, Bode J. Multiplexing RMCE: versatile extensions of the Flprecombinase-mediated cassette-exchange technology. J Mol Biol. 2010;402:52-69.

Turan S, Zehe C, Kuehle J, Qiao J, Bode J. Recombinase-mediated cassette exchange (RMCE) - a rapidly-expanding toolbox for targeted genomic modifications. Gene. 2013;515:1-27.

Typas A, Nichols RJ, Siegele DA, Shales M, Collins SR, Lim B, et al. High-throughput, quantitative analyses of genetic interactions in E. coli. Nat Methods. 2008;5:781-7.

van der Ploeg JR. Analysis of CRISPR in Streptococcus mutans suggests frequent occurrence of acquired immunity against infection by M102-like bacteriophages. Microbiology. 2009;155:1966-76.

Wagner EG, Flardh K. Antisense RNAs everywhere? Trends Genet. 2002;18:223-6.

Wang B, Kuramitsu HK. Inducible antisense RNA expression in the characterization of gene functions in Streptococcus mutans. Infect Immun. 2005;73:3568-76.

Wang BL, Ghaderi A, Zhou H, Agresti J, Weitz DA, Fink GR, et al. Microfluidic high-throughput culturing of single cells for selection based on extracellular metabolite production or consumption. Nat Biotechnol. 2014;32:473-8.

Wang H, Yang H, Shivalila CS, Dawlaty MM, Cheng AW, Zhang F, et al. One-step generation of mice carrying mutations in multiple genes by CRISPR/Cas-mediated genome engineering. Cell. 2013a; $153: 910-8$.

Wang HH, Huang PY, Xu G, Haas W, Marblestone A, Li J, et al. Multiplexed in vivo His-tagging of enzyme pathways for in vitro single-pot multienzyme catalysis. ACS Synth Biol. 2012;1:43-52.

Wang HH, Isaacs FJ, Carr PA, Sun ZZ, Xu G, Forest CR, et al. Programming cells by multiplex genome engineering and accelerated evolution. Nature. 2009;460:894-U133.

Wang YH, Wei KY, Smolke CD. Synthetic biology: advancing the design of diverse genetic systems. Annu Rev Chem Biomol Eng. 2013b;4:69-102.

Warner JR, Reeder PJ, Karimpour-Fard A, Woodruff LB, Gill RT. Rapid profiling of a microbial genome using mixtures of barcoded oligonucleotides. Nat Biotechnol. 2010;28:856-62. 
Way JC, Collins JJ, Keasling JD, Silver PA. Integrating biological redesign: where synthetic biology came from and where it needs to go. Cell. 2014;157:151-61.

Winzeler EA, Shoemaker DD, Astromoff A, Liang H, Anderson K, Andre B, et al. Functional characterization of the $S$. cerevisiae genome by gene deletion and parallel analysis. Science. 1999;285:901-6.

Wood AJ, Lo TW, Zeitler B, Pickle CS, Ralston EJ, Lee AH, et al. Targeted genome editing across species using ZFNs and TALENs. Science. 2011;333:307.

Xiao H, Zhao H. Genome-wide RNAi screen reveals the E3 SUMO-protein ligase gene SIZ1 as a novel determinant of furfural tolerance in Saccharomyces cerevisiae. Biotechnol Biofuels. 2014;7:78.

Young JJ, Cherone JM, Doyon Y, Ankoudinova I, Faraji FM, Lee AH, et al. Efficient targeted gene disruption in the soma and germ line of the frog Xenopus tropicalis using engineered zinc-finger nucleases. Proc Natl Acad Sci U S A. 2011;108:7052-7.

Yu BJ, Kang KH, Lee JH, Sung BH, Kim MS, Kim SC. Rapid and efficient construction of markerless deletions in the Escherichia coli genome. Nucleic Acids Res. 2008;36:e84.

$\mathrm{Yu}$ H, Gerstein M. Genomic analysis of the hierarchical structure of regulatory networks. Proc Natl Acad Sci U S A. 2006;103:14724-31.

Zaslaver A, Bren A, Ronen M, Itzkovitz S, Kikoin I, Shavit S, et al. A comprehensive library of fluorescent transcriptional reporters for Escherichia coli. Nat Methods. 2006;3:623-8.

Zhang Y, Buchholz F, Muyrers JP, Stewart AF. A new logic for DNA engineering using recombination in Escherichia coli. Nat Genet. 1998;20:123-8.

Zhang YX, Perry K, Vinci VA, Powell K, Stemmer WP, del Cardayre SB. Genome shuffling leads to rapid phenotypic improvement in bacteria. Nature. 2002;415:644-6. 


\section{Figure captions}

1149 Fig. 1. Overview of various genome-scale engineering tools. (A) A circular donor cassette can

1150 be integrated into the recognition site by an integrase. By flanking a target sequence with 1151 heterospecific sites, RMCE enables replacement of a target sequence using a donor cassette 1152 flanked by compatible sites. (B) The ss-oligos containing designed mutations are incorporated 1153 into the lagging strand of replicating DNA through recombineering. (C) HR or NHEJ is greatly 1154 promoted via DSBs using various endonucleases. (D) Site specific insertion can be achieved via 1155 group II introns. (E) Transcription factor (TF) libraries can be constructed by mutating the 1156 endogenous TFs (gTME) or introducing artificial TFs for large-scale perturbation on 1157 transcriptome. (F) Different regulatory non-coding RNAs (ncRNAs), including sRNAs, siRNAs 1158 and gRNAs, are used to modulate targeted gene expression in bacteria and yeast.

Fig. 2. Genome-wide strain libraries for high-throughput genotyping. Single mutations can

1161 be introduced through plasmid-borne libraries or directed genome editing. A double-mutation 1162 strain library can be created using the synthetic genetic array (SGA) method, whereby a query 1163 strain harboring the first mutation can be mated with a strain library to incorporate a genome1164 wide second mutation.

Fig. 3. Reporter based phenotyping. In the presence of a ligand, the specific activation of 1167 transcription factor (TF) is controlled by the expression of the reporter gene. A synthetic 1168 riboswitch acts as a biosensor for desired metabolites. 
1170 Fig. 4. Multiplex automated genome engineering (MAGE) and trackable multiplex 1171 recombineering (TRMR) accelerated $\boldsymbol{E}$. coli genome evolution. MAGE enables rapid 1172 generation of sequence diversity via continuous delivery of ss-oligos into cells. With barcode 1173 incorporated oligos, TRMR enables simultaneous creation and tracking of multiple genetic 1174 modifications.

1176 Fig. 5. RNAi-assisted genome evolution (RAGE) in S. cerevisiae. In the presence of a 1177 heterologous RNAi pathway, genome-wide knockdown screening can be performed with a 1178 double-stranded RNA library derived from genomic DNA. Iterative RNAi screen may help to 1179 accumulate beneficial genetic modifications in an evolving yeast genome for continuous 1180 improvement of a complex phenotype. 
1182 Fig. 1.

A

$$
\text { Recombinase }
$$

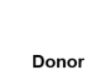

Target

Edited genome

D

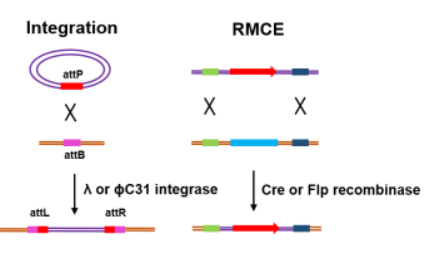

Group II introns

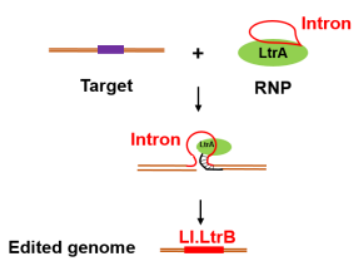

B

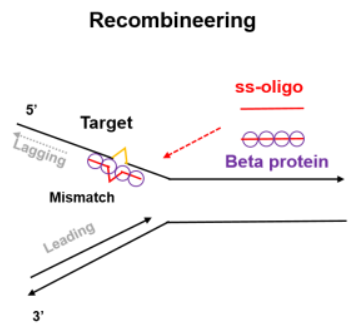

E

Transcription factor library

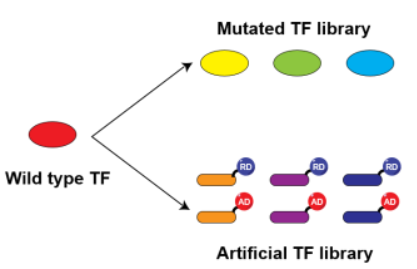

C

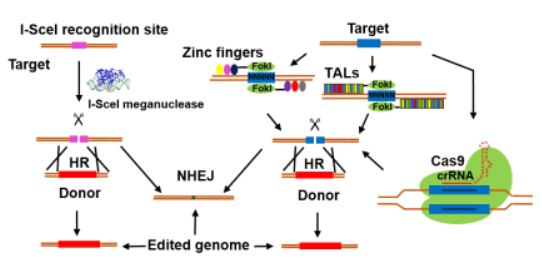

F

Regulatory ncRNAs

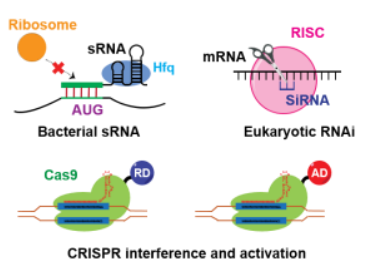

1184

1185 
Fig. 2.
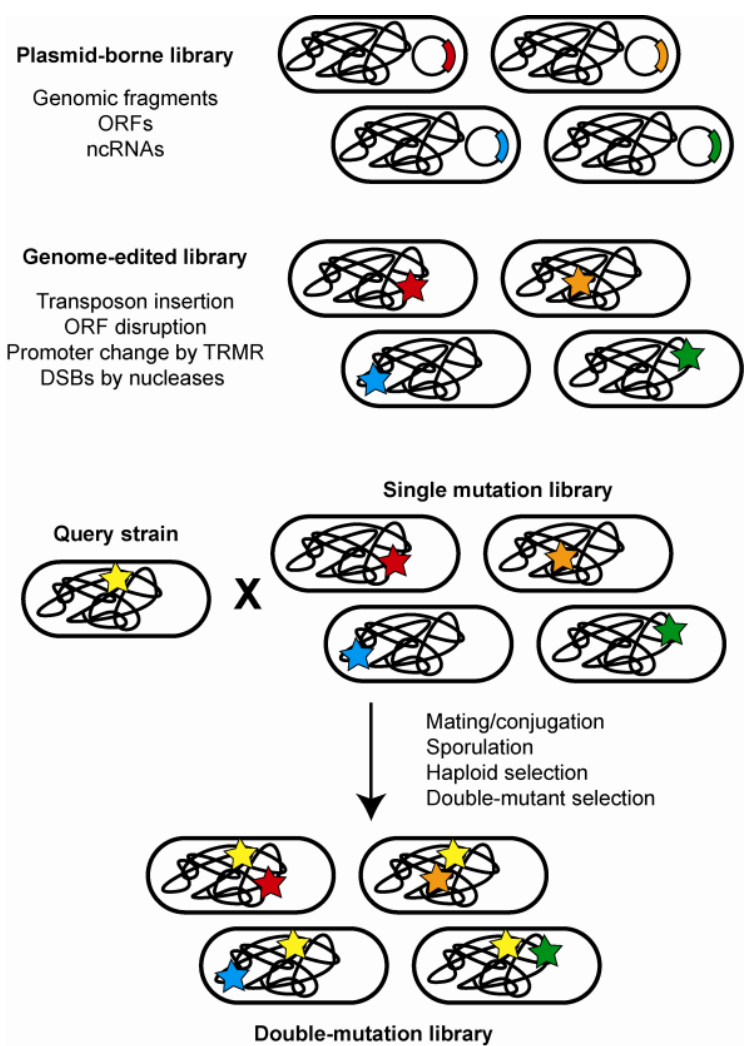
1189 Fig. 3.

TF-promoter pair

Synthetic riboswitch

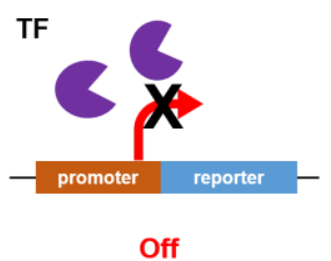

Off
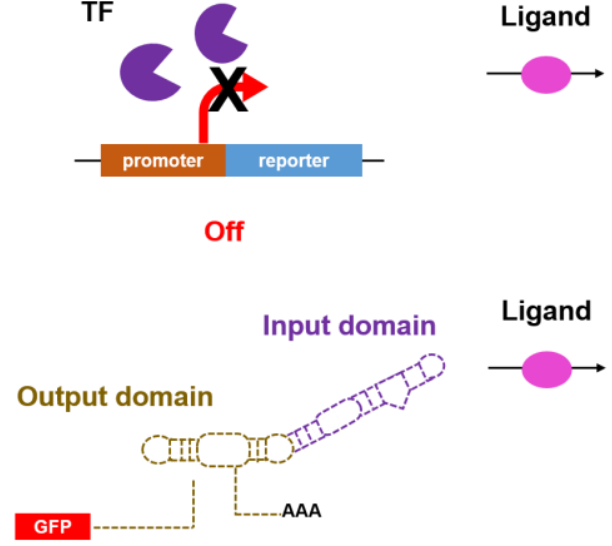

1190

Off

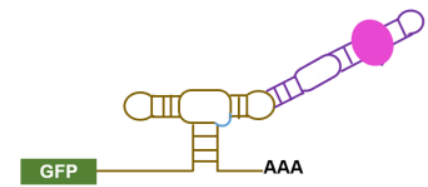

On 
1192 Fig. 4
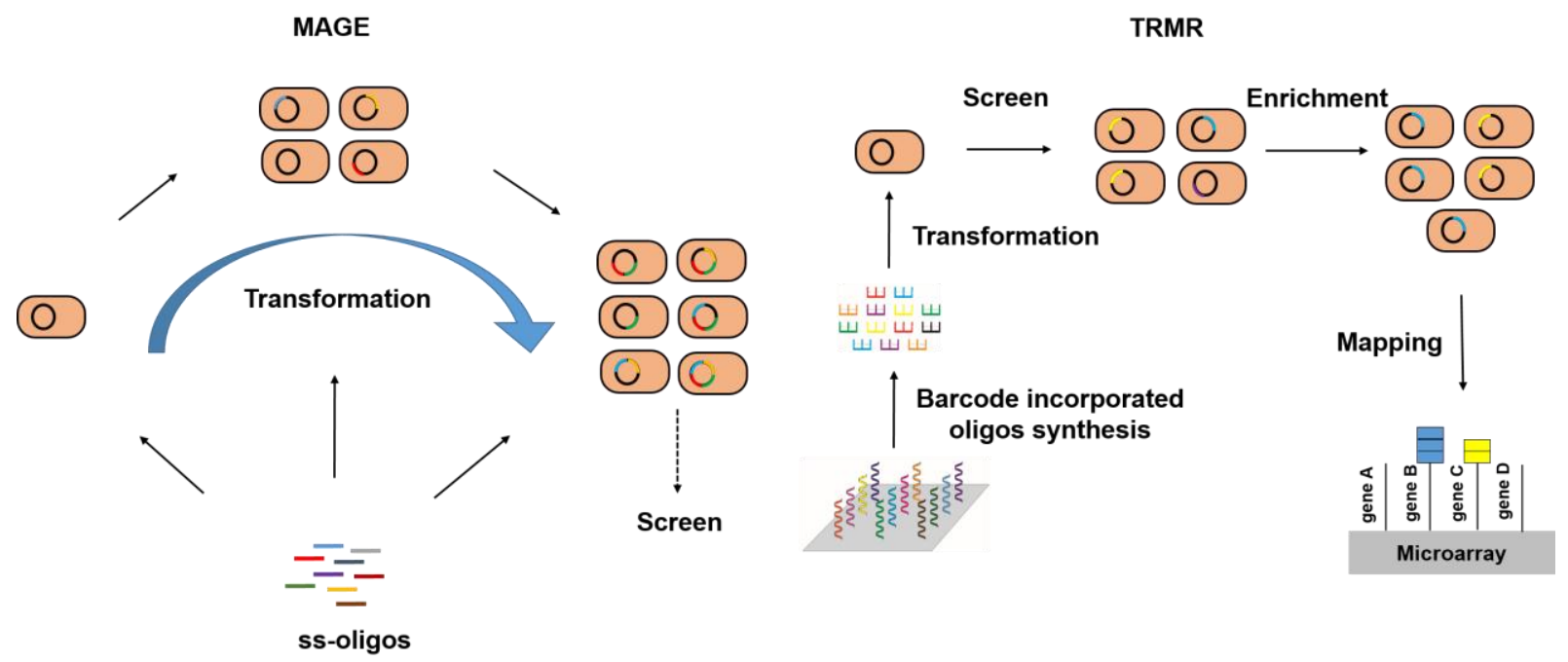
1194 Fig. 5

RNAi screen in S. cerevisiae

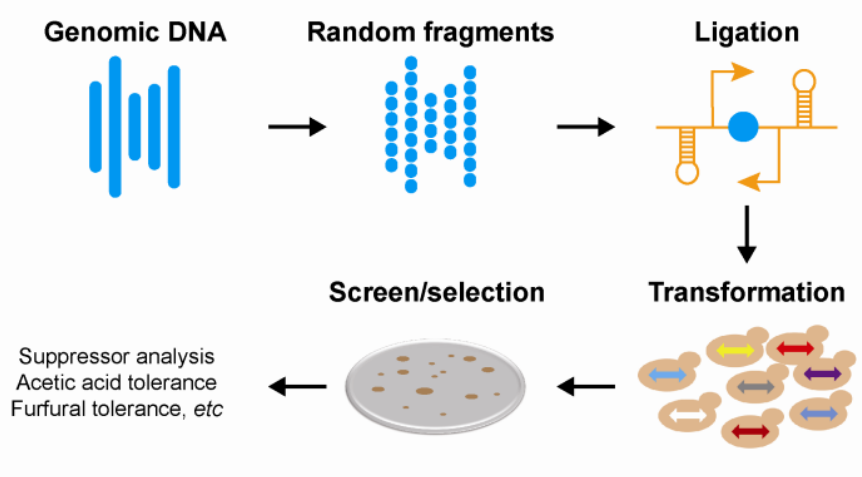

RNAi-assisted genome evolution (RAGE)

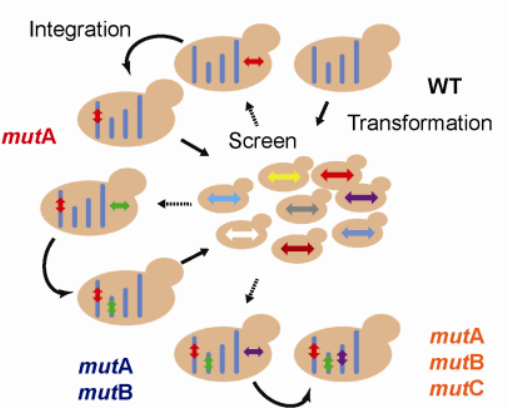

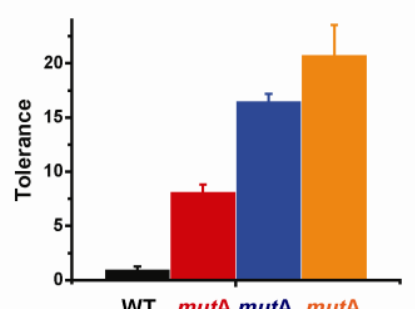

WT mutA mutA mutA mutB mutB 